\title{
Body-Centric Terahertz Networks: Prospects and Challenges
}

\author{
Nasir Saeed, Senior Member, IEEE, Mohamed Habib Loukil, Student Member, IEEE, \\ Hadi Sarieddeen, Member, IEEE, Tareq Y. Al-Naffouri, Senior Member, IEEE, \\ and Mohamed-Slim Alouini, Fellow, IEEE
}

\begin{abstract}
Following recent advancements in Terahertz (THz) technology, $\mathrm{THz}$ communications are currently being celebrated as key enablers for various applications in future generations of communication networks. While typical communication use cases are over medium-range air interfaces, the inherently small beamwidths and transceiver footprints at $\mathbf{T H z}$ frequencies support nano-communication paradigms. In particular, the use of the THz band for in-body and on-body communications has been gaining attention recently. By exploiting the accurate $\mathrm{THz}$ sensing and imaging capabilities, body-centric $\mathrm{THz}$ biomedical applications can transcend the limitations of molecular, acoustic, and radio-frequency solutions. In this paper, we study the use of the THz band for body-centric networks, by surveying works on THz device technologies, channel and noise modeling, modulation schemes, and networking topologies. We also promote THz sensing and imaging applications in the healthcare sector, especially for detecting zootonic viruses such as Coronavirus. We present several open research problems for body-centric $\mathbf{T H z}$ networks.
\end{abstract}

Index Terms-THz communications, body-centric networks, channel modeling, modulation, coding, networking, sensing, imaging, Coronavirus

\section{INTRODUCTION}

As the deployment of the fifth-generation (5G) of wireless mobile communications is in full swing, the research community is moving forward, looking into beyond $5 \mathrm{G}$. The common topic across different research projects is the usage and applications of higher frequencies in the millimeterwave (mmWave) and terahertz (THz) frequency bands. While mmWave communications are already defining 5G [1], [2], THz-band communications are expected to play a vital role in the upcoming sixth-generation (6G) wireless technology [3] [22]. Therefore, THz-related research has attracted significant funding and standardization efforts are already underway [23][25].

The $\mathrm{THz}$ band between the microwave and optical bands is the only piece of the radio-frequency (RF) spectrum that remains unutilized for communication purposes. Therefore,

Nasir Saeed is with the Department of Electrical Engineering, National University of Technology, Islamabad, Pakistan (Email: mr.nasir.saeed@ieee.org)

Mohamed Habib Loukil, Hadi Sarieddeen, Tareq Y. Al-Naffouri, and Mohamed-Slim Alouini are with the Department of Computer, Electrical and Mathematical Sciences and Engineering (CEMSE), King Abdullah University of Science and Technology (KAUST), Thuwal, Makkah Province, Kingdom of Saudi Arabia, 23955-6900 (email: mohamedhabib.loukil@kaust.edu.sa; hadi.sarieddeen@kaust.edu.sa; tareq.alnaffouri@kaust.edu.sa; slim.alouini@kaust.edu.sa). This work was supported by the KAUST Office of Sponsored Research. both the microwave and optical bands' wireless communication technologies are expected to stretch to support $\mathrm{THz}$ communications. From the RF communications perspective, $\mathrm{THz}$ frequencies start with $100 \mathrm{GHz}$, below which is the mmWave band. From the optical communication perspective, $\mathrm{THz}$ frequencies are below $10 \mathrm{THz}$ (the far-infrared) (see Fig. 11. According to the IEEE 802.15 Terahertz Interest Group (IGthz), the THz range is $300 \mathrm{GHz}-10 \mathrm{THz}$ [26].

Besides enabling high-speed wireless communications, the $\mathrm{THz}$ band has numerous medical applications, such as in characterizing tablet coatings, investigating drugs, dermatology, oral healthcare, oncology, and medical imaging [27]. Another promising use of the $\mathrm{THz}$ band is to enable connectivity in body-centric networks (BCNs). Due to the unique $\mathrm{THz}$ propagation properties and sensing capabilities, and due to the THz radiation's relative safety on biological tissues [28], the $\mathrm{THz}$ spectrum can improve the performance of existing $\mathrm{BCNs}$, thus enabling various medical applications [29]. The BCN paradigm consists of connected bio-nano sensors and devices that compute various physical and physiological phenomena, such as heart rate and glucose level, as shown in Fig. 2. These networks can operate inside the human body in real-time for health monitoring and medical implant communication [30]. Also, BCNs can feature in e-drug delivery systems [31].

$\mathrm{BCNs}$ can further be classified into two categories, onbody and in-body networks (OBNs and IBNs). OBNs utilize wearable devices or implanted nano-sensors that provide continuous monitoring of patients. OBN devices principally use the radio-frequency $(\mathrm{RF})$ technology to disseminate information; however, the main disadvantages of this technology are high-energy consumption and electromagnetic interference. On the contrary, IBNs consist of tiny-devices, also called nano-machines, that patrol within the body and collect critical health-related information [32]. Primitively, natureinspired molecular communication is used to connect such nano-machines [33]. Other technologies are investigated for BCN applications such us acoustic solutions [34], [35]. Furthermore, researchers are currently investigating a wide range of electromagnetic frequencies [36], [37], where the optical and $\mathrm{THz}$ bands are considered as promising alternatives. In addition, there are some attempts to use hybrid systems for communicating inside the human body [38], [39]. To better understand different $\mathrm{BCN}$ paradigms, the interested readers are referred to [31], [32] that briefly discuss these wireless technologies for nano communications. In Table I, we enlist the literature on promising wireless technologies for IBNs. 


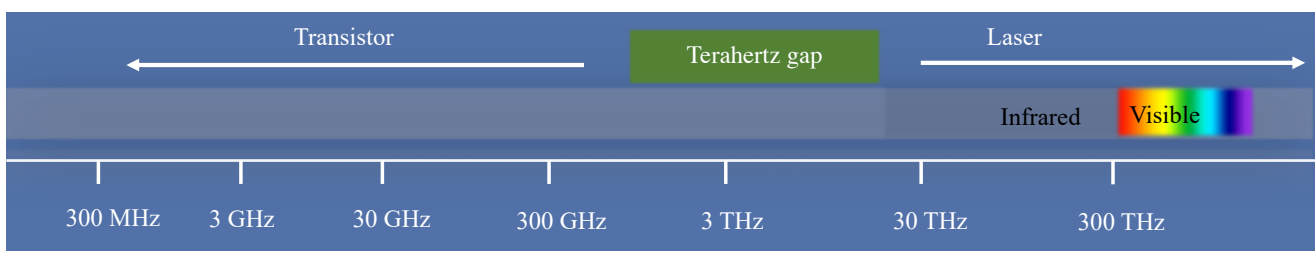

Fig. 1: The THz gap.

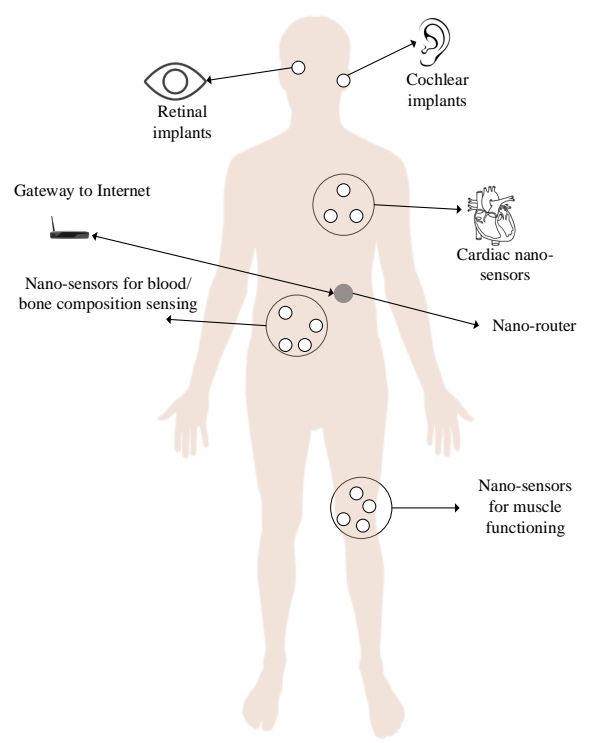

Fig. 2: Illustration of $\mathrm{BCN}$ architecture with nano-scale devices.

Inter-connecting the nano-devices to form a network that can exchange information and achieve a common goal is vital and challenging in BCNs. Since nano-devices are quite different from conventional sensing devices, the communication technologies that interconnect them need to be re-evaluated.
The most obvious solution seems to be RF technologies; however, nano-devices' distinct properties require modifying the existing channel models, communication protocols, and networking architectures. Nevertheless, the RF technology is not suitable for BCNs due to its lack of compactness, high complexity, and power consumption [29]. Therefore, recent research has shown that the $\mathrm{THz}$ band has the potential to enable low complexity, power-efficient, and high-bandwidth point-to-point links in BCNs [32].

$\mathrm{THz}$ communications still face various challenges, such as high path loss due to atmospheric losses and spreading, limiting their communication range [129]. For example, to communicate at distances of a few millimeters, the entire $\mathrm{THz}$ band is available due to negligible atmospheric loss. However, to establish links in the order of meters without the support of additional antenna and power gains, only sub$\mathrm{THz}$ frequencies in the order of tens of gigahertz $(\mathrm{GHz})$ can be used. Furthermore, direct single-hop long distances are difficult to achieve with $\mathrm{THz}$ frequencies due to the high attenuation. As a result of such constraints, novel THz-specific transceiver designs are required, that should be of compact size, high-sensitivity, low-power, and low-noise figure. Carbon nanotubes [130], complementary metal-oxide-semiconductor (CMOS), [131], silicon-germanium (BiCMOS) [132], and graphene-based [133] transceivers are potential candidates.

Connecting the nano-devices in BCNs is thus crucial, and can be achieved using various wireless communication technologies. For OBNs, there are multiple options to connect wearable devices, such as RF, optical, and $\mathrm{THz}$ paradigms.

TABLE I: Literature on promising wireless technologies for IBNs.

\begin{tabular}{|c|c|c|c|c|c|}
\hline \multirow{2}{*}{ Issues addressed } & \multicolumn{3}{|c|}{ Electromagnetic } & \multirow{2}{*}{ Molecular } & \multirow{2}{*}{ Acoustic } \\
\hline & RF & THz & Optical & & \\
\hline Channel modeling & $[40]-\mid 56]$ & 57-66 & [67|-|71] & 33 , $72-78$ & $\begin{array}{l}[34], \mid \overline{35}] \\
{[\overline{79}],[\overline{80}]}\end{array}$ \\
\hline Noise modeling & $[46], \mid 56],[81]$ & {$[\overline{58}],[\overline{82}]-[\overline{87} \mid$} & {$[67]$} & {$[88]-[91]$} & {$[35]$} \\
\hline Performance analysis & $\begin{array}{c}{[41],[46],[56]} \\
\quad[92],[93]\end{array}$ & {$\left[\frac{59}{[83},|,| \frac{86}{94}\right]$} & [67]-[71] & $\begin{array}{c}{[\overline{72}],[\overline{95}]} \\
{[91],[96 \mid-[98]}\end{array}$ & [35], |79] \\
\hline $\begin{array}{l}\text { Experimental } \\
\text { demonstration }\end{array}$ & [81], |93], |99| & |100], [94] & - & {$[101],[102]$} & {$[80]$} \\
\hline $\begin{array}{l}\text { Modulation and } \\
\text { coding schemes }\end{array}$ & |56] & [28], [94], [103]-[105] & [68|, |69], |106| & {$[107 \mid-[112]$} & $\begin{array}{c}{[79 \mid,[80]} \\
{[113]}\end{array}$ \\
\hline Networking & {$[114 \mid$} & |115|-|117] & [70] & $\begin{array}{c}{[\overline{74} \mid,[\overline{78}]} \\
{[118]}\end{array}$ & $\begin{array}{c}{[79 \mid,[80]} \\
{[119]}\end{array}$ \\
\hline Security & & {$[120],[121]$} & & {$[120]-[123$} & [120], [121] \\
\hline Testbeds & [92], [124]-[126] & {$[62|| 63],,[100 \mid$} & - & $\begin{array}{c}{[127],[128]} \\
77]\end{array}$ & [79], |113| \\
\hline
\end{tabular}


However, in IBNs, the possibilities are limited to molecular and $\mathrm{THz}$ communications, due to the aforementioned limitations of in-vivo RF communications [134]. Hence, the research on $\mathrm{THz} \mathrm{BCNs}$ is promising where novel transceiver designs, antennas, channel models, modulation and coding schemes, and networking topologies are still in progress. Since several research challenges still face $\mathrm{THz} \mathrm{BCNs}$, it is crucial to review these networks' various aspects and highlight promising research directions. Towards this end, this tutorial aims at outlining the physical and network layer considerations for implementing $\mathrm{THz} \mathrm{BCNs}$. This tutorial also highlights the importance of $\mathrm{THz}$ sensing and imaging in biomedical applications, especially for detecting zootonic diseases such as the Coronavirus disease (COVID-19).

\section{A. Related Surveys}

Several surveys address $\mathrm{THz}$ communications in general, providing insights on both the device and communication aspects [3]-[8], [22], [135]. The communication aspects mainly target channel modeling paradigms, signal processing techniques, novel routing protocols, and supporting experimentation. There are also a few dedicated review articles that discuss $\mathrm{THz}$ communications for nano-networks (NNs). Among these is the pioneering work of Akyildiz et al. [136], that discusses various possibilities of communication in $\mathrm{NNs}$, and introduces the architecture for nano-networks, consisting of nano-nodes, nano-routers, and micro/nano-interfaces. Similar insights can be found in [137], with a better focus on molecular communications. The work in [138] further surveys the literature on big data analytics for future healthcare systems. Moreover, [135] discusses the medium access control (MAC) layer protocols for NNs and compares the performance of various MAC protocols in terms of transmission distance, collision probability, and energy consumption.

Several other contributions [139]-[143] target different aspects of NNs, converging at a common idea of two possible communication options, namely, molecular and $\mathrm{THz}$ communications. The former is more suitable for IBNs, whereas $\mathrm{THz}$ can be used for both IBNs and OBNs. Among these contributions, [142] is more relevant to our work, as it summarizes various requirements of IBNs, including functional (purpose of communication), technical (reliability, security, safety, etc.), and legal (e.g., implantability of the devices and duration of the body contact) aspects. Table $\mathrm{II}$ summarizes these relevant articles.

\section{B. Contributions of this Paper}

In the context of the surveys mentioned above, we can see that most of the contributions are outdated. i.e., dating back to 2016, except [22], [135], [138] and [146], which are more generic, focusing on the $\mathrm{THz}$ band and its usage for future wireless communication networks. Therefore, these recent works do not focus on the prospects of the $\mathrm{THz}$ band for body-centric networks. Hence, we believe that it is quite important to articulate a tutorial on $\mathrm{THz}$ communications for BCNs, which can help both academia and industry. Unlike the existing works, our tutorial focuses on the critical aspects
TABLE II: Comparison of relevant review articles.

\begin{tabular}{|c|c|c|}
\hline Ref. & Year & Area of Focus \\
\hline Akyildiz et al [3] & 2010 & $\begin{array}{l}\text { Presents } \mathrm{THz} \text { communications for } \mathrm{NNs} \text {, } \\
\text { channel modeling, and routing protocols }\end{array}$ \\
\hline Jornet et al. $[\overline{137}]$ & 2012 & $\begin{array}{l}\text { Introduces the internet of multimedia nano- } \\
\text { things (IoMNT), a possible architecture for } \\
\text { IoMT, and networking protocols }\end{array}$ \\
\hline $\begin{array}{l}\text { Rikhtegar et al. } \\
{[144]}\end{array}$ & 2013 & $\begin{array}{l}\text { Studies molecular and electromagnetic } \\
\text { communications, nano-devices, and net- } \\
\text { working for NNs }\end{array}$ \\
\hline $\begin{array}{l}\text { Balasubramaniam } \\
\text { et al. } \mid[139]\end{array}$ & 2013 & $\begin{array}{l}\text { Presents various applications of NNs and } \\
\text { how to connect the NNs with the outside } \\
\text { world }\end{array}$ \\
\hline $\begin{array}{l}\text { Akyildiz et al. [3], } \\
\text { [4] }\end{array}$ & 2014 & $\begin{array}{l}\text { Focuses on } \mathrm{THz} \text { applications at the nano } \\
\text { and macro levels and presents possible } \mathrm{THz} \\
\text { band transceivers and antennas }\end{array}$ \\
\hline Miraz et al. $|\overline{141}|$ & 2015 & $\begin{array}{l}\text { Overviews connecting the NNs with the } \\
\text { internet of things (IoT) and its applications }\end{array}$ \\
\hline $\begin{array}{l}\text { Dressler et } a l . \\
{[142]}\end{array}$ & 2015 & $\begin{array}{l}\text { Focuses on healthcare applications using } \\
\text { in-body NNs and presents various network- } \\
\text { ing architectures }\end{array}$ \\
\hline Petrov & 2016 & $\begin{array}{l}\text { Presents an overview of the } \mathrm{THz} \text { band for } \\
\text { future wireless communication networks }\end{array}$ \\
\hline tal. $\mid \overline{138}]$ & 2018 & $\begin{array}{l}\text { Focuses on big data analytics, such as data } \\
\text { gathering, processing, feature extraction, } \\
\text { and predictive modeling for healthcare ap- } \\
\text { plications }\end{array}$ \\
\hline $\begin{array}{l}\text { Ghafoor et al. } \\
{[135]}\end{array}$ & 2019 & $\begin{array}{l}\text { Presents applications of the } \mathrm{THz} \text { band and } \\
\text { various MAC layer protocols for THz com- } \\
\text { munications }\end{array}$ \\
\hline Lemic et al. $\mid \overline{146}]$ & 2019 & $\begin{array}{l}\text { Focuses on THz communications for NNs } \\
\text { and its applications }\end{array}$ \\
\hline $\begin{array}{l}\text { Sarieddeen et al. } \\
{[22]}\end{array}$ & 2020 & $\begin{array}{l}\text { Presents an overview of } \mathrm{THz} \text { communi- } \\
\text { cation, sensing, imaging, and localization } \\
\text { along with their applications }\end{array}$ \\
\hline This & 2020 & $\begin{array}{l}\text { Focuses on the issues of } \mathrm{THz} \text { devices, } \\
\text { communications, and networking for THz- } \\
\text { band body centric networks, highlighting } \\
\text { various applications in the health industry }\end{array}$ \\
\hline
\end{tabular}

of THz-based BCNs, including device technologies, channel and noise modeling, modulation and networking algorithms, and applications in the healthcare industry. Moreover, we also present the latest research highlighting that THz-band communications can help in pandemic response management by detecting the Coronavirus disease (COVID-19). We further present various future research challenges for THz-band BCNs and their applications.

\section{Organization}

The rest of this tutorial is organized as follows. In Section II we present an overview of the $\mathrm{THz}$ signal sources and antennas, such as electronic, photonic, integrated hybrid devices, and graphene-based $\mathrm{THz}$ antennas. Section III presents the current research work on various communications technologies for $\mathrm{BCNs}$, focusing more on $\mathrm{THz}$ band channel modeling for IBNs. Section IV then covers various modulation schemes for THz-based IBNs. Moreover, in Section $\mathrm{V}$, we discuss numerous network topologies and routing protocols for BCNs. Section VI further highlights the $\mathrm{THz}$ band's importance for sensing and imaging applications, including detection of COVID-19. Then, in Section VII, we illustrate the latest consensus on the health effects of $\mathrm{THz}$ radiation. Finally, in Section VIII, we focus on future research challenges for THz- 
based BCNs, followed by Section IX, which concludes this tutorial.

\section{THz Signal Sources, Antennas, And Detectors}

The main contributions to $\mathrm{THz}$ technology are still at the device level rather than the system level. High-frequency electromagnetic radiation is perceived as waves that are treated via electronic devices (the mmWave realm) or as particles that are processed via photonic devices (the optical realm). Novel solutions are considering the use of both electronic and photonic materials for $\mathrm{THz}$ transceiver design.

On the electronic side, silicon-based systems [147], [148] that have been used for mmWave applications are being pushed for $\mathrm{THz}$ communication usage. Typical solutions include silicon complementary metal-oxide-semiconductor (CMOS) and silicon-germanium (SiGe) BiCMOS technologies [131], [132], [149], [150], which demonstrate incredible compactness and compatibility with existing fabrication processes. Furthermore, higher operating frequencies have been noted using heterojunction bipolar transistors (HBTs) [151], [152], III-Vbased semiconductors [153], high electron mobility transistors (HEMTs) [154]-[156], and Schottky diodes [157]. In [150], SiGE-HBT devices are shown to operate at $720 \mathrm{GHz}$. Other experimental demonstrations with different frequencies and transmission powers are highlighted in [158]-[161].

The main design driver for photonic solutions, on the other hand, is data rate [162], where higher carrier frequencies are supported, but the degrees of integration and output power are both low. Frequencies beyond $300 \mathrm{GHz}$ have been supported using optical downconversion systems [162], quantum cascade lasers [163], photoconductive antennas [164], and uni-traveling carrier photodiodes [165]. The use of resonant tunneling diodes (RTDs) is also shown to deliver oscillation frequencies up to $2 \mathrm{THz}$ [166]-[169]. Moreover, hybrid photonic-electronic solutions are also emerging [170]. These developments have improved the generation, modulation, and radiation of $\mathrm{THz}$ waves [162], [170]-[172]. While photonic devices can be used for OBNs, their prospect use in IBNs is more challenging due to their relatively bulky structures.

The use of plasmonic materials for $\mathrm{THz}$ communications is also gaining popularity [129], [173]. In particular, graphene is considered the best choice for nano-antennas that support EM nano communications, high electron mobility, and reconfigurability [174]-[177]. Plasmonic solutions are capable of directly generating waves in the true $\mathrm{THz}$ range in much more compact (order of $\mu \mathrm{m}$ ) and flexible designs, by supporting the propagation of surface plasmon-polariton (SPP) waves [178]. For instance, [179] proposes a $\mathrm{THz}$ transceiver made of graphene that can be utilized for nano communications. In fact, graphene can be used to develop direct $\mathrm{THz}$ signal sources, modulators (that manipulate amplitude, frequency, and phase), and on-chip THz antenna arrays [180]. Graphenebased designs are further favored for IBN applications for their low electronic noise temperature and their ability to generate short pulses that save energy [103].

\section{Channel AND Noise Modeling}

As a pre-requisite for understanding $\mathrm{THz}$ technology's role in $\mathrm{BCN}$ applications, it is essential to investigate the propagation and noise models of $\mathrm{THz}$ transmissions inside the human body. This section summarizes the studies performed to characterize the in-body $\mathrm{THz}$ channel and noise models, taking into account several phenomena such as molecular absorption caused by human tissues, spreading resulting from the expansion of the wave, and reflection and scattering of signals. By taking these factors into account, we shape a comprehensive understanding of $\mathrm{THz}$ BCNs that defines the achievable propagation distances, assists in performance analysis, and predicts communications scenarios.

\section{A. Channel Modeling}

Being the pioneers to introduce the concept of $\mathrm{THz}$ transceivers for nano-communications [29], Akyildiz et al. introduced the channel model for $\mathrm{THz}$ propagation in the air [129], which was later adopted by the research community for different applications. In this work, we illustrate how IBNs can benefit from nano-scale components operating in the $\mathrm{THz}$ band, where transmissions are less vulnerable to propagation losses, especially scattering [181]. Since the propagation medium for IBNs is the human tissue, it is vital to understand this medium by acquiring its parameters before modeling the propagation at $\mathrm{THz}$ frequencies. Building on multiple studies on $\mathrm{THz}$ channel modeling over the air [129], [182], the works in [59], [60], [183] adopt a similar propagation loss model for modeling in-body $\mathrm{THz}$ channels.

The path loss in human skin consists of two components: absorption and spreading losses [59]. In [83], [183], [184], the IBN path loss is modeled using the modified Friis equation as

$$
\operatorname{PL}(f, d)[d B]=\operatorname{PL}_{\mathrm{abs}}(f, d)[d B]+\mathrm{PL}_{\mathrm{spr}}(f, d)[d B],
$$

where $\mathrm{PL}_{\mathrm{spr}}$ denotes the spread loss due to the expansion and the propagation of the wave in the medium, $d$ is the total length of the path, $f$ is the operating frequency, and $\mathrm{PL}_{\mathrm{abs}}$ represents the absorption loss. The spreading loss can be further expressed as

$$
\operatorname{PL}_{\text {spr }}(f, d)[d B]=20 \log _{10}\left(\frac{4 \pi d n_{r}}{\lambda_{0}}\right),
$$

where $n_{r}$ represents the real part of the refractive index of the medium and $\lambda_{0}=c / f$ is the free-space wavelength. The absorption loss in (III-A) is expressed as

$$
\mathrm{PL}_{\mathrm{abs}}(f, d)[d B]=10\left(\mu_{\mathrm{abs}}\right) d \log _{10}(e),
$$

where the absorption coefficient $\mu_{\mathrm{abs}}$ and the refractive index are obtained from measurements.

We can also derive these parameters from the complex dielectric parameters of the human skin, in particular, the complex permittivity, $\epsilon=\epsilon_{r}-j \epsilon_{i}$, and the complex refractive index, $n=n_{r}-j n_{i}$, where $j$ denotes the complex number $\left(j^{2}=-1\right)$ and the subscripts $r$ and $i$ represent the real and imaginary parts, respectively. Note that the parameters $\mu_{\mathrm{abs}}, \epsilon$, $\epsilon_{r}$, and $\epsilon_{i}$ are frequency dependent. The following equations 


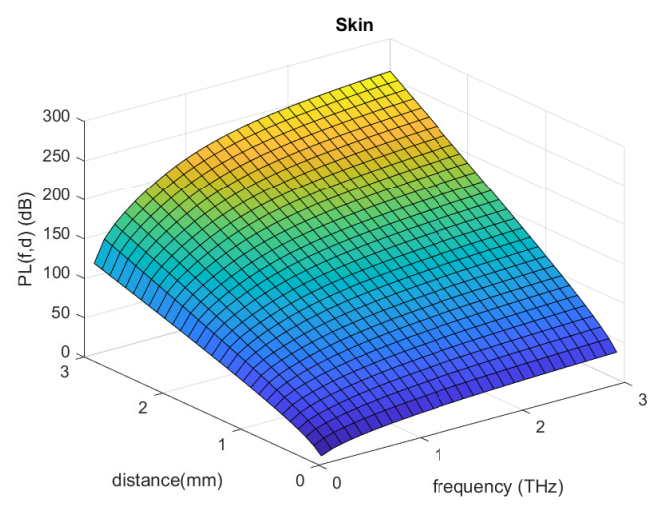

(a) Skin path loss

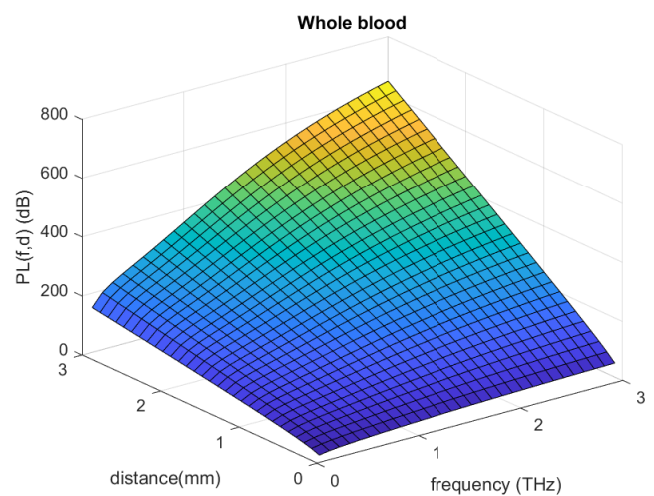

(b) Whole blood path loss

Fig. 3: Skin and whole blood path loss as function of distance and frequency.

describe the relations between the electromagnetic and optical parameters

$$
\begin{aligned}
\mu_{\mathrm{abs}} & =\frac{4 \pi n_{i}}{\lambda_{0}}, \\
\epsilon & =n^{2}, \\
\epsilon_{r} & =n_{r}^{2}-n_{i}^{2}, \\
\epsilon_{i} & =2 n_{r} n_{i} .
\end{aligned}
$$

Another way to compute the absorption coefficient is to model it from individual particles [58]. This procedure is complicated because there are various elements with different concentrations in the human body. A simpler alternative in [58], [61], [64] uses complex permittivity, which is computed using the double Debye relaxation model [185]. The technical report in [186] gives more insight into different models, describing the absorption coefficient and refractive index by performing several experiments.

Since the human body is mainly composed of water and sodium chloride $(\mathrm{NaCl})$ solutions, [100] investigates the path loss model for these components. The analytical and simulation results are found to almost agree, where it is shown that $\mathrm{THz}$ communications are possible in water over a millimeter range. Using [III-A], [100] computes the total path loss for different human tissues. Intuitively, the increase in communication distance or frequency leads to an increase in path loss. Also, the path loss varies for different tissues for a given frequency and distance. For example, the path loss in the blood is higher compared to skin and fat. This is due to the water concentration difference, with the highest percentage of water being in the blood. The work in [61] further investigates the absorption coefficient and refractive index for human blood in the $\mathrm{THz}$ band, where experiments are conducted on different blood components: Full blood, plasma, blood cells, and blood clots, showing their different responses alongside pure water. Due to the heavy existence of water molecules, plasma and whole blood encounter prominent path losses. The simulation in Fig. 3 demonstrates that the path loss is higher in blood cells compared to skin tissues for a given distance and frequency. Similarly, [62], [63] study the use of $\mathrm{THz}$ spectroscopy on artificial human skin (Dermis) that mainly consists of blood vessels and hair cells, by characterizing the human tissue in terms of absorption coefficients and refractive indices as a function of $\mathrm{THz}$ frequencies.

A THz pulsed spectroscopy (TPS) method is proposed in [64] to study the $\mathrm{THz}$ refractive index and absorption coefficient of the human skin. The authors investigate the accuracy of the measurements compared to the known data, where agreements are noted with healthy human skin, except for some body parts; in-body $\mathrm{THz}$ propagation depends on the structure and thickness of each component of the skin. The analysis in [64] takes into consideration the built-in noise in experimental waves to solve the TPS inverse problem. It also excludes the examination of lower and higher frequencies due to the fluctuation of error at these frequency ranges (because of different scattering on the surface and in the tissue volume). Moreover, [66] examines $\mathrm{THz}$ propagation inside the human body by considering a multi-layered human tissue model, where the components of the cascaded multi-layer intrabody model are, from deep to superficial, blood, fat, skin, and air. To better characterize the communication link between the nanodevices inside and outside the body at $\mathrm{THz}$ band, [66] proves the need to account for cross-layer reflection.

A recent IBN channel model that also considers the scattering effect of the $\mathrm{THz}$ waves alongside the absorption and spreading loss is proposed in [58], where

$$
\operatorname{PL}(d, f)[d B]=\mathrm{PL}_{\mathrm{abs}}[d B]+\mathrm{PL}_{\mathrm{spr}}[d B]+\mathrm{PL}_{\mathrm{sca}}[d B] .
$$

The scattering loss is caused by small and large particles, and the corresponding loss factor is expressed as

$$
\mathrm{PL}_{\mathrm{sca}}[d B]=10\left(\mu_{\mathrm{sca}}^{\mathrm{small}}+\mu_{\mathrm{sca}}^{\text {large }}\right) d \log _{10}(e),
$$

where $\mu_{\mathrm{sca}}^{\text {small }}$ and $\mu_{\mathrm{sca}}^{\text {large }}$ are the absorption coefficients due to small and large particles, respectively. The scattering factor can be ignored in IBNs since it is negligible compared to the absorption coefficient.

Despite that the previous path loss models are the most popularly used models for IBNs, several researchers have proposed simpler models, and we hereby distinguish two main alternatives. The first is an analytical path loss model which 
TABLE III: THz-band IBN channel models.

\begin{tabular}{|c|c|c|c|}
\hline Equation & Type & Focus & Ref. \\
\hline III-A & Analytical & $\begin{array}{l}\text { Accounts for molecular ab- } \\
\text { sorption and spreading. }\end{array}$ & $|\overline{59}| \overline{183}$ \\
\hline III-A & Analytical & $\begin{array}{l}\text { Accounts for molecular ab- } \\
\text { sorption, spreading and scat- } \\
\text { tering. }\end{array}$ & $\mid 58$ \\
\hline (III-A) & Empirical & Log distance path loss model. & |57 \\
\hline III-A & Empirical & $\begin{array}{l}\text { A correlation-based model } \\
\text { which takes into account } \\
\text { distance, frequency alongside } \\
\text { number of ducts. }\end{array}$ & 60 \\
\hline
\end{tabular}

is expressed in [57] as

$$
\operatorname{PL}(d, f)[d B]=10 \log _{10}(K)+10 n \log _{10}\left(\frac{d}{d_{0}}\right)+X_{\sigma},
$$

where $K$ and $n$ are the path loss at reference distance $d$ and path loss exponent, respectively, and $X_{\sigma}$ represents the shadowing effect caused by particles in the path, which is modeled as a random variable following the log-normal distribution. The second alternative model is empirical, based on actual measurements for IBNs in the THz band [60]. An artificial layer that mimics the human tissue is used to perform multiple experiments, resulting in the path loss model

$$
\operatorname{PL}(d, f)[d B]=A(N)+B(N) r^{0.65}+C(N) f^{4.07},
$$

where $A(N), B(N)$, and $C(N)$ are the offset coefficient, distance coefficient, and frequency coefficient, respectively. These coefficients are a function of the number of sweat ducts $N$, which is explained by the fact that they are mainly composed of water, causing higher absorption at $\mathrm{THz}$ frequencies; a regression-based technique is used to obtain these coefficients. We summarize the channel models used for THz-band IBNs in Table III

\section{B. Noise Modeling}

Besides the path loss, $\mathrm{THz}$ systems encounter a noise factor that originates from different sources. The main noise component comes from molecular noise. According to [84], [86], the molecular noise is composed of two parts: Body radiation noise and molecular absorption noise. During wave propagation inside the human body, a fraction of the energy is absorbed, causing an increase in the temperature. This phenomenon is considered as noise, namely body radiation noise or atmospheric noise, where the medium becomes an effective black body radiator. This background noise can be represented using the sky noise model and is a function of the transmission medium's temperature. The corresponding noise temperature is expressed as

$$
T_{\mathrm{mol}}=T_{0}\left(1-e^{-\mu_{\mathrm{abs}} d}\right),
$$

where $\mu_{\mathrm{abs}}$ is the absorption coefficient and $T_{0}$ is the reference temperature. This noise model is more suitable for communications in the atmosphere. As highlighted in [84], the use of temperature as a parameter is not reliable for evaluating noise inside the human tissues. Therefore, in line with the work in [82], the work in [84] uses the following expression of background noise (which is given using Plank's law):

$$
B\left(T_{0}, f\right)=\frac{2 h \pi(n f)^{3}}{c^{2}}\left(e^{\frac{h f}{k_{B} T_{0}}}-1\right)^{-1},
$$

where $B\left(T_{0}, f\right)$ is the Plank's function, $n$ is the corresponding refractive index of the $\mathrm{THz}$ wave in the human tissue, and $h$ and $k_{B}$ are the Plank's and Boltzmann's constants, respectively. Consequently, the power spectral density of the background noise inside the human body is expressed as

$$
N_{b}(f)=B\left(T_{0}, f\right) \frac{c^{2}}{4 \pi\left(n_{0} f_{0}\right)^{2}} .
$$

Alongside the propagation waves' attenuation, the molecules in the medium introduce molecular absorption noise due to internal vibration caused by the incident $\mathrm{THz}$ waves. As a result, this would transform into retransmission of EM radiation at the same frequency in random directions [187]. This phenomenon is considered to result in an additional noise factor for intra-body communications. In [28], [59], this noise is represented using the same model in [129]. The corresponding power spectral density (PSD) can be defined as

$$
N_{m}(f, d)=k_{B} T_{0}\left(1-e^{\frac{4 \pi}{c} f d \kappa}\right),
$$

where $\kappa$ is the extinction coefficient. Based on [82], the work in [84] expresses the PSD as

$$
N_{m}(f, d)=S_{T x}(f)\left(\frac{c}{4 \pi n f d}\right)^{2}\left(1-e^{-\mu_{\mathrm{abs}} d}\right),
$$

where $S_{T x}(f)$ is the PSD of the transmitted signal. Finally, the total noise PSD is given by

$$
N(f, d)=N_{m}(f, d)+N_{b}(f) .
$$

There are other noise models for IBNs in the literature. For instance, in [83], the noise temperature for the $\mathrm{THz}$ band is modeled as the sum of three different noises: $T_{\mathrm{sys}}$, originating from the electronic system, $T_{\text {other }}$, emerging from other surrounding devices, and a third one coming from molecular absorption $T_{\mathrm{mol}}$, and is expressed as

$$
T_{\mathrm{mol}}=T_{0}\left(1-e^{\frac{4 \pi}{c} f d \kappa}\right) .
$$

The total noise can be expressed as a sum of the three components:

$$
T_{\text {noise }}=T_{\text {mol }}+T_{\text {sys }}+T_{\text {other }} \text {. }
$$

Note that in graphene-based systems, the electronic noise temperature is quite low and can be neglected, leaving only the molecular absorption noise temperature as a main contributor to the overall noise [188].

Another approach for IBN noise modeling is presented in [87]; it considers a stochastic noise consisting of three parts: the Johnson-Nyquist noise, the black-body noise, and the Doppler-shift-induced noise. The overall noise PSD is derived after figuring out each component's probability distribution, verified via 2-D particle simulations. 


\section{Modulation Schemes}

Even though THz-band communications offer an extra piece of spectrum, the design of adequate $\mathrm{THz}$ modulation schemes remains critical for efficient utilization of this new spectrum, especially that we encounter multiple unsolved constraints in transceivers' conception. Generally, at high-frequency communications, carrier-modulated signals use low-complexity modulation schemes to mitigate the technical restrictions in the design of $\mathrm{THz}$ modulators. According to [6], THz modulators do not follow the capabilities of THz transmission. Despite the considerable progress in $\mathrm{THz}$ modulators, their speed does not exceed a few gigabits-per-second (Gbps), which hinders the use of complex modulations for $\mathrm{THz}$ communication systems. Such constraints have motivated researchers to think about new optimized modulation techniques for $\mathrm{THz}$ applications.

While the achievable data rate may not be of primary concern in IBNs, the communication paradigms should be energy efficient. Nanosensors cannot communicate using classical communication techniques of long duration signals due to the size and energy constraints, especially when implanted inside the human body. Given the availability of large bandwidths, short pulses are considered for intra-body $\mathrm{THz}$ communications. The work in [103] introduces the transmission of pulses in an asymmetric On-Off Keying modulation Spread in Time (TS-OOK). The TS-OOK technique uses a hundred femtosecond-long pulse for binary " 1 ", and silence for logical " 0 ". To avoid confusion of silence from no transmission with the transmission of logical " 0 ", the authors propose taking advantage of initialization preambles and constant-length packets. Therefore, the transmitted signal can be expressed as

$$
s(t)=\sum_{k=1}^{K} A_{k} p\left(t-k T_{s}\right),
$$

where $K$ is the number of transmitted bits, $A_{1}, A_{2}, \ldots A_{K}$ are the amplitudes of the transmitted symbols where each $k$ th transmitted symbol $A_{k}$ can take the values 0 and $1, p(t)$ denotes the Gaussian pulse with duration $T_{p}$, and $T_{s}$ is the time between two consecutive pulses where $T_{p}<<T_{s}$.

Due to the correlation between communication capabilities and the distribution of the transmitted power in the frequency domain, three types of in-body communications paradigms can be distinguished:

- Flat communication: It assumes that the total transmitted power is uniformly distributed over the entire operative bandwidth.

- Pulse-based communication: It considers pulses that can be modeled as Gaussian waves, especially with the promising use of graphene for $\mathrm{THz}$ communications.

- Optimal communication: Given that the channel is frequency selective, the total bandwidth is divided into small, locally flat sub-bands. The power is then allocated as a function of frequency-selective properties of the medium to maximize the overall channel capacity.

These communication types are analyzed in [28], [94] by highlighting the channel capacity and communication ranges of each. It is noted that optimal communication outperforms the flat and pulsed alternatives in both channel capacity and transmission ranges, as it can adapt its power distribution to the attenuation levels experienced at multiple frequencies. The results also show that the transmission range cannot exceed $9 \mathrm{~mm}$, which inspires designing effective networking techniques like multi-hop communications alongside new MAC and routing protocols. However, pulse-based modulations can be argued to be more beneficial in THz IBNs. In addition to their simplicity, pulse-based modulations can mitigate the noise effect. For instance, in the case of graphene-based transceivers, the electronic noise is negligible, and the source of noise is molecular absorption only, which emerges from the re-emissions of the absorbed radiation. Such noise only affects the pulses and not the silences. In [116], TS-OOK is utilized to propose a realistic network architecture and communication scheme for in-vivo $\mathrm{THz}$ communications assuming a human hand scenario.

Single-pulse variable duration (SPVD) modulation [104] is another novel modulation scheme that can be tailored for THzband IBNs. It is a combination of TS-OOK and pulse position modulation (PPM), which aims at ensuring higher energy efficiency rather than achieving high data rates. This novel scheme is based on varying the single-pulse duration, which reduces the energy consumption of nanosensors. In particular, a fixed number of bits are transmitted using a waveform that is composed of a certain number of silences and a very short pulse; the latter is in the order of 100 femtoseconds. The proposed design of SPVD waveforms consists of a variable number of equal time slots with a variable number of silences and one slot for the pulse. Consider $M=2^{k}$ to be the modulation order, where $k$ is the number of bits per symbol. Then, each vector of different bitstreams corresponds to a unique transmitted waveform which is expressed as

$$
s_{n}(t)=p\left(t-n T_{\text {slot }}\right), \quad t \leq(n+1) T_{\text {slot }},
$$

where $p(t)$ is a 100 -femtosecond-long pulse, $n \in$ $\{0,1, \ldots, M-1\}$ denotes the number of silences in the waveform, and $T_{\text {slot }}$ is the time slot duration. SPVD proves to be more efficient than classical TS-OOK, especially that it manages to send more information by using fewer pulses and more silences. Taking into account that the main source of noise is molecular absorption caused by re-radiated waves, this approach reduces the effect of noise and thus increases the overall signal-to-noise ratio. Therefore, compared to TSOOK, SPVD sends the same information using less energy, but at the expense of longer time durations. As previously mentioned, such a delay may not be of primary concern for $\mathrm{BCNs}$ operating at $\mathrm{THz}$ frequencies.

\section{Networking Topologies}

Besides channel modeling and modulation techniques, effective networking in BCNs is crucial because nanoscale devices suffer from many limitations. In the following, we summarize some of these challenges:

- The nanoscale size of $\mathrm{BCN}$ devices limits their processing and memory capabilities. One way to overcome the energy constraint is to use energy-harvesting techniques at a nanoscale level, by converting fluidic, vibrational, 
and EM energies into electrical energy [189]. Moreover, BCNs need to consider the balance between energy consumption and harvesting. Also, the nanoscale components limit the devices' battery size that further limits the amount of stored energy.

- Another major hurdle for nanoscale networking is the use of flooding mechanisms in which each node broadcasts packets blindly, thus leading to severe collisions and redundancy.

- As discussed in the previous sections, the $\mathrm{THz}$ band suffers from molecular absorption, spreading, and scattering losses. The substantial path loss limits the overall efficiency of the network.

- The limited memory of nanoscale $\mathrm{THz}$ devices is also a challenge for multi-hop transmission, especially in $\mathrm{THz}$ networks where the transmitter and receiver use directional communication [190].

To overcome these challenges, $\mathrm{BCNs}$ require appropriate network layer protocols that can enable an efficient path for communicating among various nanodevices under various network constraints [191]. The networking protocols mainly aim to maximize the network lifetime, improve coverage and reliability, reduce latency, and minimize energy consumption. In the following, we review various networking protocols for BCNs that can be classified into single- and multi-path routing protocols.

\section{A. Single-Path Routing (SPR)}

The routing protocols which consist of a single path between the transmitter and the receiver consume less energy than multi-path routing protocols. However, SPR can lead to a low packet delivery rate and high latency. Optimal SPR protocols should consume less power, minimize the delay, and improve the packet delivery rate. In the following, we discuss various protentional SPR protocols for BCNs.

1) Energy-saving Routing (ESR): As nanodevices are implanted on the human body in BCNs, a hierarchical architecture is required to maintain an efficient wireless communication link. Accordingly, [192] proposes an energy-saving routing (ESR) protocol for $\mathrm{THz}$-operating $\mathrm{BCNs}$, in which the main components of the BCNs consist of various nanodevices, including nano-sensors (NSs), nano-cluster controllers (NCCs), and a nano-network controller (NNC). In ESR, the network is divided into multiple layers based on the NS's distance from the NNC and their single-hop transmission range (see Fig. 4p. Once the layers are defined, the NSs with leftover residual energy are selected as NCCs. Then, the NCCs broadcast an advertisement, and each NS picks its NCC based on the received signal strength indicator (RSSI) (joining the NCC with the highest RSSI value). The information from the NS to the NNC is transmitted through the NCC by using a time division multiple access protocol. This multi-layered clustering and time division approach reduces data collision and the network's overall energy consumption. However, one major issue with the ESR protocol is that it allows only oneor two-hop transmission, thus requiring a dense deployment of NSs and NCCs.

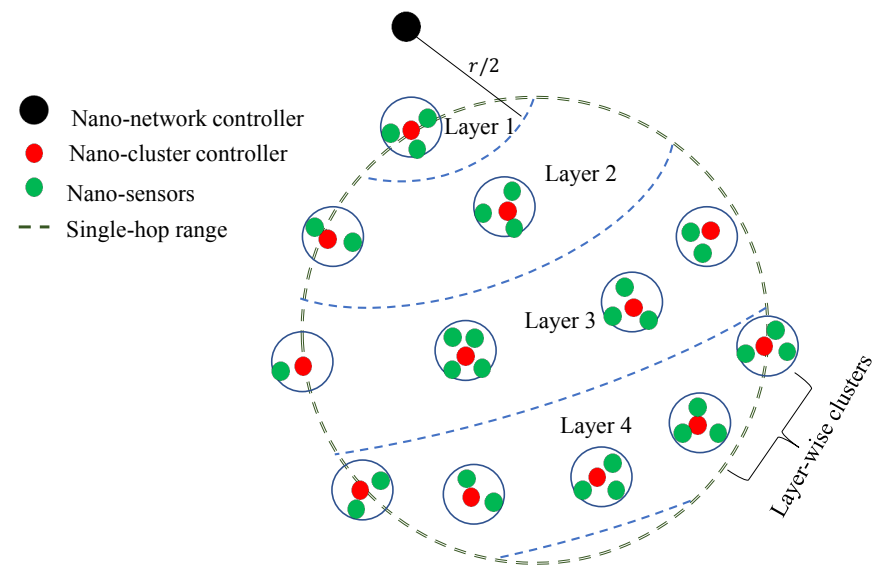

Fig. 4: ESR protocol: The NNC is placed at the center of all layers, whereas the link between the NCC and NS is singlehop.

2) Multi-hop Decision Algorithm (MHDA): Another potential routing protocol for THz-based BCNs is the multihop decision algorithm (MHDA) that ensures infinite network lifetime while achieving a sufficient throughput [115]. Similar to ESR, the framework of MHDA is hierarchical, consisting of NSs and NCCs. In general, the NCCs have more resources than the NSs, and therefore act as cluster heads. The MHDA estimates the probability of the amount of average energy consumed in single- and multi-hop transmissions. It suggests that when the power of each NS is optimized, the multihop transmission can result in lower energy consumption. The expression for the probability of energy saved for any arbitrary $\mathrm{SN} i$ in a multi-hop transmission is given as

$$
P_{e}(i)=\operatorname{Pr}\left\{E_{b m}(i) \leq E_{b d}(i)\right\},
$$

where $E_{b m}(i)$ and $E_{b d}(i)$ are the average energies required for a single bit in a multi- and single-hop transmissions, respectively. Moreover, $P_{e}(i)$ depends on the density of NSs and the distance $\left(d_{i}\right)$ between the $i$ th NS and the NNC. Consider that the distance between $i$ th NS and its random neighbor is $d_{i n}$, and the distance between the $i$ th NS and an NNC is $d_{i c}$, which are both random variables and follow a Poisson point process (see Fig. 5). Then, $P_{e}(i)$ can be expressed as

$$
P_{e}(i)=1-e^{\rho A\left(d_{i c}\right)}
$$

where $\rho$ is the NS density and $A\left(d_{c}\right)$ is the spanning area to find the neighboring NS. Moreover, in MHDA, the NS adjusts its transmission power for selecting the next hop based on its residual energy and multi-hop transmission.

3) Energy-efficient Routing (EER): In the energy-efficient routing (EER) protocol, the calculations are mainly performed by the NNC to reduce the computation burden on NSs [193]. One significant difference between MHDA and EER is that in the latter, the NS stores the distance between itself and the $\mathrm{NNC}$, where the single-hop range of the NS is kept fixed, reducing the computational complexity. In the EER protocol, different regions are defined based on the distance between NS 


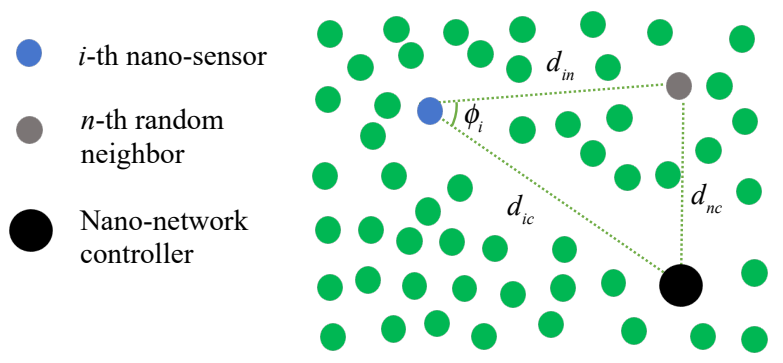

Fig. 5: Illustration of single- and multi-hop transmission in MHDA. The probability of having less energy consumption through multi-hop path $\left(d_{i n}-d_{n c}\right)$ is higher than using the direct path $\left(d_{i c}\right)$.

and NNC and the single-hop range of the NS, as shown in Fig 6 For instance, the regions $\mathrm{A} 1$ and $\mathrm{A} 2$ are within the singlehop range of the source NS, whereas region A3 is defined by the radial distance between the source NS and NNC. The EER protocol consists of the following steps:

- The NNCs broadcast hello signals to which the NSs respond and share their location and ID.

- When the $i$ th NS wants to send data, it checks whether the NNC is in its single-hop range or not. If the NNC is in single-hop range, the $i$ th NS can directly send the data to the NNC; however, if the NNC is not in a single-hop range, the $i$ th NS broadcasts query signals to neighbor NSs.

- The NSs in region A2 becomes the candidate set of nodes, where each candidate calculates its link cost and sends it to the source NS. Once the $i$ th source NS receives all the neighbors' responses, it selects the forwarding node based on the smallest link cost. This process continues until the $\mathrm{NNC}$ receives the data.

One major advantage of the EER protocol is that it limits the number of forwarding candidates by taking into account the direction towards the NNC. Both the energy consumption and computation complexity of EER is less than MHDA.

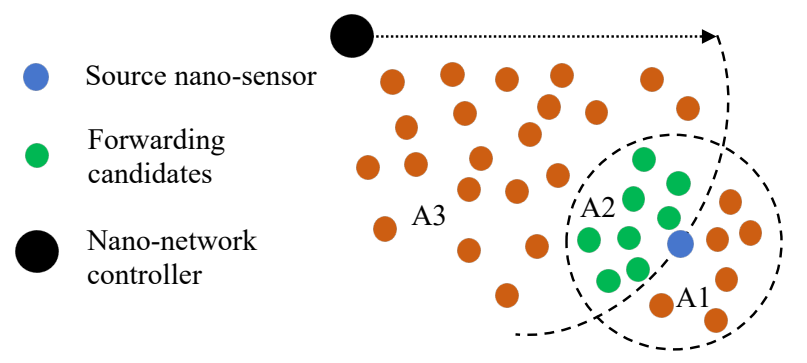

Fig. 6: Illustration of path selection in EER protocol. Candidate forwarding nodes are defined based on single-hop distance from the source and direction towards the nanonetwork controller.

4) Time-to-live-based Routing (TTLR): Unlike the data tier network in THz-based BCNs that consists of densely populated NSs, the backhaul tier is formed by sparse NNCs.
Therefore, the dynamic channel in the THz-band leads to higher vulnerability to the backhaul tier. To compensate for this issue, [194] proposes a low complexity, time-to-live-based routing (TTLR) scheme for NNCs that adapts itself to the dynamic channel state. In the TTLR protocol, NNCs collect data from NSs and forward it to the IoT gateway, where the aim is to select a few NNCs among all in the presence of a dynamic channel. First, the IoT gateway disseminates a beacon signal for polling to extract the data. The packet in the polling signal sets the time-to-live (TTL) for beacons to a maximum value. The forwarding path is then decided based on the total number of neighboring NNCs for each NNC and the number of NNCs accumulated during the transmission. To further elaborate, consider five NNCs as shown in Fig. 7. here "NNC 5" selects "NNC 2" as a forwarding node instead of "NNC 4" because the cumulative number of neighbors for "NNC 2" is less than "NNC 3". One major problem of the TTLR protocol is that it does not take into account the energy of NSs, which is a critical factor for nano-scale devices in $\mathrm{BCN}$.

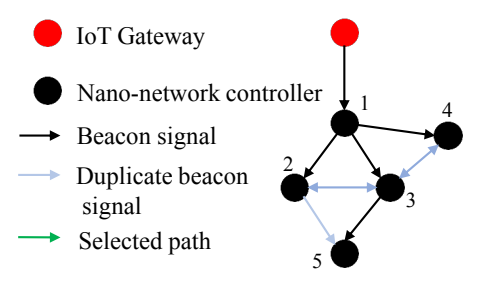

Polling signal broadcast

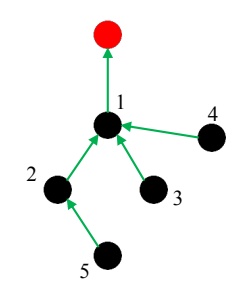

Path selection
Fig. 7: Illustration of TTLR protocol; on the left side, we have a polling mechanism while the right figure shows the selected path.

5) Probability-based Routing (PBR): A low-energy consumption probability-based routing (PBR) protocol for BCNs is proposed in [195]. The PBR protocol assumes that the NSs can only communicate with the NCC due to their limited capabilities, thus forming a hierarchical architecture. The path selection in PBR depends on the probability of NCC existence, which further depends on the density of NCCs. The PBR protocol consists of four steps: Topology detection, probability distribution, decision, and data forwarding. An external entity mainly maintains the topology detection, whereas the probability distribution is calculated based on density and area of the clusters and cells. The routing path is then selected based on the probability distribution of the cells rather than the shortest route. As PBR's routing metric is based on the probability of having NCCs in a specific cell, it has better end-to-end reliability than the shortest path.

\section{B. Multi-Path Routing (MPR)}

Unlike SPR, multi-path routing is based on a flooding mechanism that allows all the NSs to forward data, leading to a higher number of transmissions and collisions and increasing the total energy consumption. There are mainly two techniques used in MPR: Area-based limiting-flooding (ALF) 
and infrastructure-based dynamic-flooding (IDF). Only limited NSs are selected as candidate nodes in ALF protocols to forward the packets from the source to the destination. On the contrary, based on the received packet quality, the NSs are classified as "infrastructure" or "users" in IDF protocol, where the NSs are defined as infrastructure forward the data. In the following, we discuss various potential MPR protocols for BCNs.

1) RADAR Routing: A simple flooding-based routing protocol (RADAR) for THz nano-networks is introduced in [196], in which the NSs are distributed equally in a circular region with an NNC at the center. The NSs, which are in the transmission beam of the NNC, are in on-state while the rest of the NSs are in off-state, reducing the total energy consumption. Also, the packets are only transmitted in a sectorbased approach leading to controlled flooding. Nevertheless, in RADAR, a routed packet may be lost as the receiving NS may be in an off-state. In RADAR, the probability of the receiving NSs to be in on-state is correlated with the beamwidth rotation of the transmission signal. For example, a flexible beamwidth rotation can lead to a large number of on-state NSs, increasing the overall energy consumption and coverage with a higher number of packet collisions. On the contrary, limited beamwidth rotation leads to a higher packet delivery ratio and reduced energy consumption. Therefore, it is crucial to optimize the beamwidth for the RADAR routing protocol. Due to the RADAR protocol's simplicity, it can be a potential candidate for routing in BCNs.

2) CORONA Routing: Another popular energy-efficient network layer protocol for THz-based wireless nano-networks is coordinated-and-routing (CORONA) [197]. In CORONA, the NSs are uniformly deployed in a rectangular region where four beacon NSs are positioned at the corner of the area (see Fig. 87. Each beacon node transmits a signal based on which all the NSs compute their hop counts to each beacon and define their coordinates. Once the NS coordinates are defined, flooding is performed by the NSs which are located between the source NS and the destination NS. For example, if "NS A" (near to beacon A) wants to transmit a packet to "NS B" (near to beacon B), then the NSs in the blue region can only re-transmit the packets until it arrives at "NS B". The NS coordinates are defined based on the hop-counts from the beacons where flooding is limited to a specific region. CORONA has proved to be an energy-efficient point-to-point routing protocol for nano-networks, which makes it well-suited for BCNs.

3) Stateless Linear Routing (SLR): The stateless linear routing (SLR) protocol is an extension of CORONA for threedimensional wireless nano-networks in which the information is routed through a linear path [198]. In SLR, the NSs are placed in a cubic setup with eight beacons at each corner of the cube. Similar to the CORONA protocol, in SLR, the beacons transmit a signal based on which the NSs calculate their multihop distances to each beacon. In the path selection phase, the SLR protocol selects a forwarding NS if it is positioned on a straight line between the source and the destination. To elaborate, consider that the locations of the source and destination are $x_{s}, y_{s}, z_{s}$ and $x_{d}, y_{d}, z_{d}$, respectively.

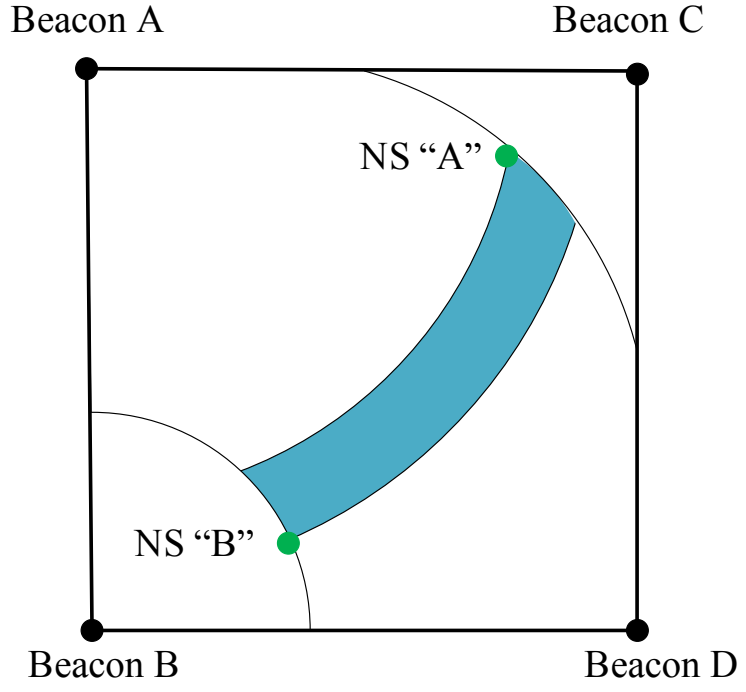

Fig. 8: Illustration of CORONA protocol by using beacons "A" and "B" for path selection. The blue area represents the possible location of forwarding nodes adopting limited flooding.

Assuming that the NS (at point $P=\{x, y, z\}$ ) is on the straight line from source to destination, then

$$
\frac{x-x_{s}}{x_{d}-x_{s}}=\frac{y-y_{s}}{y_{d}-y_{s}}=\frac{z-z_{s}}{z_{d}-z_{s}} \text {. }
$$

Since the location of SNs are based on hop counts which are integer values, V-B3 can be re-written as

$$
\begin{gathered}
\triangle_{x}=\left(x-x_{s}\right)\left(y_{d}-y_{s}\right)-\left(y-y_{s}\right)\left(x_{d}-x_{s}\right)=0, \\
\triangle_{y}=\left(x-x_{s}\right)\left(z_{d}-z_{s}\right)-\left(z-z_{s}\right)\left(x_{d}-x_{s}\right)=0 .
\end{gathered}
$$

In case the NS at $P$ is not on the straight line between the source and destination, then compensation for deviation is added to the above expressions. In general, a set of distances to three-beacon nodes can identify a specific zone, which is also referred to as a viewpoint that can be optimized to select the best viewport for a specific NS [199]. One major drawback of the SLR protocol is that each NS needs to store all eight beacons' information, which is quite large for resource-limited NSs.

4) Light-weight Self-tuning Routing (LSR): Light-weight self-tuning routing (LSR) is also a scalable and straightforward flooding-based routing protocol for wireless nano-networks [200]. The LSR protocol considers a source NS placed at the center of a square region that broadcasts the sensed data. At the packet level, there can be three possible events for an incoming packet: "PARITY_CHECK_ERROR", where the receiver collects the packet but fails the parity check, "DUPLICATION_CHECK_ERROR", where the packet is wellreceived but discarded because it was already received before, and "SUCCESS", where the packet is correctly collected for the first time. The sequence of these packet events is processed using the Misra-Gries algorithm [201], where the forwarding 


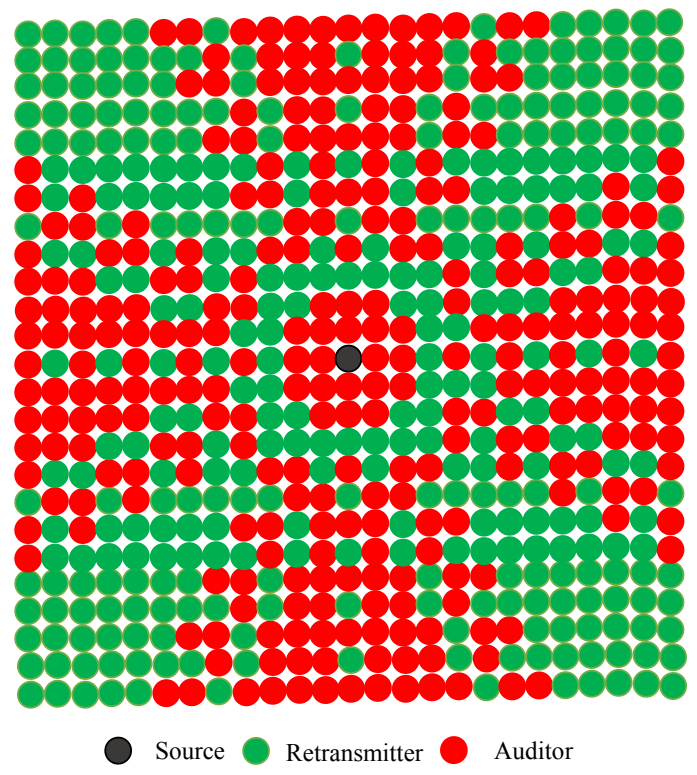

Fig. 9: Illustration of LSR protocol with a source NS, retransmitters, and passive auditors. The central NS performs sensing and transmits data to an external entity using retransmitters.

node's status is evaluated by determining the most common occurrence in the sequence, resulting in the classification of the nodes as "retransmitters' or "auditors" (see Fig. 9p. After categorization, only retransmitters blindly forward packets, while the auditors listen but do not take part in the transmission. This classification leads to the selection of straight paths from the central source NS to the external entity, reducing the latency and energy consumption. However, the LSR protocol follows a flooding-based approach, which leads to an increase in the network overhead.

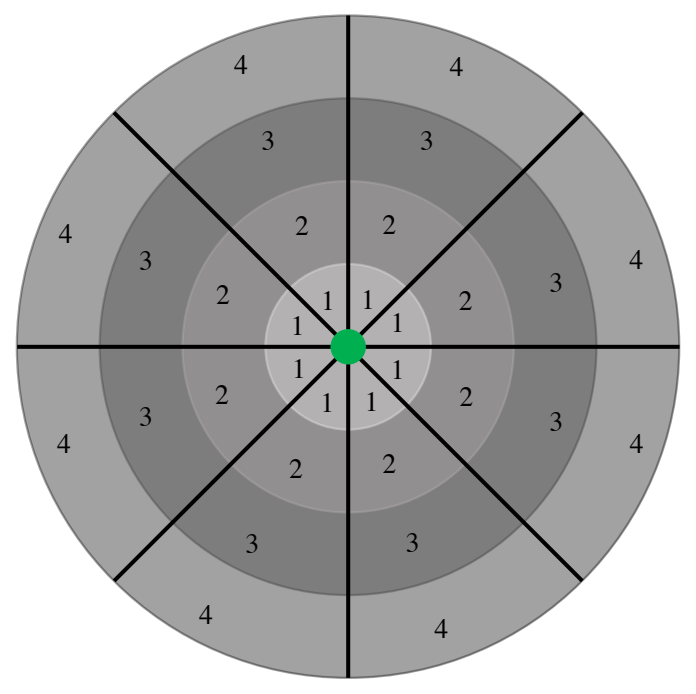

Fig. 10: Data in DR protocol is routed by using the rings of various radii and sectors.

5) Deployable Routing ( $D R)$ : Another well-known MPR for software-defined metamaterial-based wireless nano- networks is deployable routing (DR) [202]. In the DR system, NSs are spread over a circular area with a beacon at the center that periodically transmits set-up signals, based on which all other NSs define their hop counts (see Fig. 10). As in LSR, each NS computes its packet reception quality and hop count from the beacon, and then decides whether it can become a "user" or an "infrastructure". In DR routing, an NS can forward the data in two modes: (a) standard power having a large radius and (b) low-power with a small radius. After the deployment phase, the source NS "S" sends packets to the receiving NS "R" where the NS whose radius is between the source and the destination radially forwards the packets. When the radius of a forwarding node is equal to "S" or " $R$ ", it radially re-transmit the packets.

Comparison: This section covers various potential singleand multi-path network layer protocols for THz BCNs. The single-path routing protocols are energy-efficient at the cost of low reliability since they depend on a single path between the source and the destination. Furthermore, the SPR protocols follow a hierarchical architecture with NSs, NCCs, and a NNC, whereas the MPRs are based on the concept of flooding and therefore are flat in nature. Besides, some routing protocols can be more complex compared to others based on position awareness or the distance measurement to the NCCs. For instance, RADAR routing does not require any position information of the NCC, and therefore, it has low complexity, while in MHDA the NSs need to estimate the distance to the NCC, which makes it more complex. We summarize all of the above routing protocols in Table [V] where we categorize them based on their type, architecture, and performance.

\section{THz SEnsing And Imaging: A COVID-19 Use CASE}

So far, we have discussed several aspects of $\mathrm{THz} \mathrm{BCNs}$, such as signal sources, channel/noise modeling, modulation schemes, and networking aspects. Nevertheless, sensing and imaging are among the popular uses of $\mathrm{THz}-$ communications that enable various invaluable biomedical applications. This section highlights the importance of sensing and imaging in the healthcare sector and further relates it to the current COVID19 pandemic.

THz-band frequencies are currently used for various sensing and imaging applications without damaging the living tissues and bio-molecules because of their non-ionizing feature. Furthermore, research shows that many bio-molecules uniquely vibrate at $\mathrm{THz}$ frequencies, which makes this band an invaluable piece for the detection of various diseases. In general, meta-materials are used for THz-based bio-sensing; for example, [203] uses a nano-gap meta-material to detect the PRD1 and MS2 types of viruses. Moreover, THz-time domain spectroscopy (TTDS) is utilized in [204] for the detection of three Avian Influenza viruses (H1N1, H5N2, and H9N2). Their proposed $\mathrm{THz}$ nanoreflecter not only detects various types of viruses but can also be used to diagnose cancers. Generally, TTDS can operate in three modes: Transmission, reception, and reflection. The reflection-based techniques are more suited for bodies with water content, where the amount of reflected 
TABLE IV: Comparison of potential routing protocols for BCN.

\begin{tabular}{|c|c|c|c|c|c|c|c|}
\hline Protocol & Type & Location awareness & Architecture & Complexity & Scalability & $\begin{array}{l}\text { Energy- } \\
\text { efficient }\end{array}$ & Ref. \\
\hline ESR & Single-path & $\overline{\mathrm{No}}$ & Hierarchical & Medium & $\overline{\text { Good }}$ & $\overline{\text { Yes }}$ & $\overline{192}$ \\
\hline MHDA & Single-path & Distance to NCC & Hierarchical & High & Good & Yes & $\overline{115}$ \\
\hline EER & Single-path & Distance to NCC & Hierarchical & High & Good & Yes & {$[\overline{193}$} \\
\hline TTLR & Single-path & No & Hierarchical & Medium & Good & No & $\overline{194}$ \\
\hline PBR & Single-path & No & Hierarchical & Medium & Good & Yes & 195 \\
\hline$\overline{R A D A R}$ & Multi-path & No & Flat & Low & Limited & No & $\overline{196}$ \\
\hline CORONA & Multi-path & Number of hops to beacons & Flat & Low & Limited & No & {$[\overline{197}$} \\
\hline SLR & Multi-path & Number of hops to beacons & Flat & Medium & Limited & No & {$[\overline{198}$} \\
\hline LSR & Multi-path & No & Flat & Low & Good & No & 200 \\
\hline DR & Multi-path & $\begin{array}{l}\text { Hop counts to a central en- } \\
\text { tity }\end{array}$ & Flat & Low & Good & No & $\overline{202}$ \\
\hline
\end{tabular}

and scattered light from the material is used to sense the object [205]. The THz-band is thus a prolific frequencyrange for the use of spectroscopy as its vibration stimulates bio-molecules. In this context, THz-band technologies have attracted researchers to develop devices that can detect viruses, antibodies, and proteins.

Given the current COVID-19 situation, it is very timely to discuss THz-band's prospect roles in fighting this pandemic. COVID-19 is notoriously impacting humans and the overall worldwide economy. In the presence of such a zoonotic disease, it is crucial to curb the virus's spread. Various tools are introduced to slow down the spread, such as social distancing, contact tracing, and early detection. Towards this end, a fast and easy way of detecting COVID-19 is a vital and challenging task. For instance, recently, a child died in Saudi Arabia due to the break of a Coronavirus swab in his nasal cavity [206]. At the moment, the detection of COVID-19 is primarily done via polymerase chain reaction (PCR) tests. However, researchers are looking for advantageous alternative solutions. Besides the detection of COVID-19, there is also a focus on carrying out the antibody tests that can help determine a previously infected person, resulting in a better understanding of the virus spread. Researchers are looking for out-of-the-box approaches, and THz-based spectroscopy is one of the potential solutions for detecting COVID-19.

The $\mathrm{THz}$ frequencies can penetrate various materials, which can enable multiple COVID-19-related imaging-based applications. A research team at Northern Hill University suggested that THz-imaging can have a significant positive impact in low-income countries where existing thermal imaging systems may fail to detect the asymptomatic carriers at necessary places, including airports and rail stations [207]. Unlike Xrays, THz-imaging offers broader screening in populated places without obvious harmful effects on human health. THzimaging can be used to scan the upper respiratory part of the subjects to determine the difference between an infected and healthy person based on the water content in the cells, indicating the edema. One other use of THz-imaging in COVID19 detection is ex-vivo testing. For instance, researchers at the University of Negev developed a breath test that offers quick testing without disturbing the patients [208]. A patient breath sample is collected on a chip and analyzed, where $\mathrm{THz}$ resonance detects the virus particles. Although this solution is still in the clinical trial stage, it can have a broader impact on COVID-19 detection. In short, the detection of COVID-19 by using THz-imaging may become an essential application in the healthcare industry.

Besides using the $\mathrm{THz}$ band in imaging for detecting viruses, THz technology can also assist patients' remote operations during a pandemic. For example, THz-based wearable sensors or implants on the patient's body can collect health data at high rates and forward it to the healthcare support staff, where actions can be taken remotely to assist patients. This way, the crucial hospital staff will have a lower risk of contracting the virus and save the commute time.

\section{EFFeCts of Terahertz Radiation on Health}

The deployment of any new technology always raises a mixture of positive and negative feelings. Nowadays, the major focus is on $5 \mathrm{G}$ technology, and there is a strong belief among a considerable number of people that $5 \mathrm{G}$ negatively affects human health [209]. Such allegations got to a point where nonscientific communities are linking the electromagnetic fields generated by $5 \mathrm{G}$ base stations to COVID-19 [210]. These concerns triggered the work on analyzing the health risks of 5G technology. Recent papers cover the following aspects: Health effects from 5G exposure [211]-[214], health risks of $5 \mathrm{G}$ features [212], [215], 5G exposure metrics, regulations, and compliance assessment [214]-[217] and the mitigation of 5G risks [214], [217].

The work in [218] analyzes all the above aspects in a comprehensive and in-depth manner. Regarding the effects of electromagnetic waves, two classes of radiations can be distinguished:

- Ionizing radiation: These radiations have enough energy to ionize atoms of living cells (X-rays and gamma-rays are examples of this type), posing a high risk to health because exposed cells can die or become cancerous.

- Non-ionizing radiation: These radiations do not ionize living cells and thus can not result in cell death and cancer. Nevertheless, such radiation's energy can still be high enough for molecules to vibrate, raising other health concerns.

While the first class is well studied in the literature [219], the second class is further classified depending on the radiation effects as thermal and non-thermal. Authorities and organizations around the world seek to define the exposure limits to 
avoid the heating effect on the human body. However, when it comes to non-ionizing non-thermal radiation, there is no clear evidence of a correlation between $5 \mathrm{G}$ standardized radiations and the emergence of biological effects [220]-[223].

The prospect utilization of body-centric $\mathrm{THz}$ devices means that higher exposure of the human body to electromagnetic waves at much higher frequencies is expected, raising concerns about THz technology's safety. Therefore, it is necessary to understand $\mathrm{THz}$ radiation's effects on living objects and set the corresponding safety limits [224]. The $\mathrm{THz}$ spectrum is still within the range of non-ionizing radiations. In fact, $\mathrm{THz}$ radiation's low transmission energy is highly unlikely to break the chemical bonds of living cells. Similar to the above classification, we find two popular mechanisms in the literature of how $\mathrm{THz}$ waves interact with biological objects:

- Thermal effect: The strong absorption of mainly continuous $\mathrm{THz}$ waves by water leads to heating in the human body, and consequently, biological effects [225]- 227].

- Non-thermal effect: $\mathrm{THz}$ waves can generate resonance effects in DNA, which in some cases may change the molecular dynamics and damage the local hydrogen bonds between DNA strands, causing the modification of gene expression [228], [229].

Relevant papers in the literature that address the effects of $\mathrm{THz}$ radiation on biological cells treat the effect of conformation of bio-polymers (proteins and DNA) [225], [230]-[235] and discuss the organism-level responses [225], [233], [234]. Furthermore, the work in [236] investigates the effects of $\mathrm{THz}$ radiation on the cell level.

Several experiments are conducted to understand the effects of the exposure of blood, skin, and nerve cells to $\mathrm{THz}$ radiation. Different parameters are varied to explore multiple scenarios [236], including the modulation type (pulsed-based vs. continuous waves), the frequency range, the intensity of radiation, and the exposure time. Some of these demonstrations prove the effect of $\mathrm{THz}$ radiation on blood cells [237]-[241], skin cells [242]-[246], and nerve cells [247]-[249]. However, others studies did not report a negative impact on blood cells [238], [250], [251], skin cells [252], [253], and nerve cells [254]. [236] summarizes the data concerning the effects of $\mathrm{THz}$ radiation on cells; the conclusion is that $\mathrm{THz}$ radiation can indeed affect biological cells through modifications to the membranes, proliferation, and pore formation. Therefore, more in-depth studies are required to set clear rules and establish safe use limits for $\mathrm{THz}$ body-centric applications.

\section{FUTURE RESEARCH DIRECTIONS}

Recent advances in body-centric $\mathrm{THz}$ networks show the invaluable applications of this research area in the healthcare sector. However, its successful implementation is quite challenging due to many constraints, such as the tiny size of the sensors, limited energy, extreme channel conditions (human body), and complicated deployment. The research on bodycentric $\mathrm{THz}$ networks is still in the early phase, with numerous open research challenges. Therefore, in this section, we point out some of these future research directions that require further investigation.

\section{A. Development of Novel Signal Sources}

One of the major hurdles in the development of $\mathrm{THz}$ bodycentric networks $(\mathrm{BCN})$ is the availability of efficient $\mathrm{THz}$ sources. The invention of new materials (in addition to the techniques discussed in Sec. III that can support nanoscale communications can be a massive step towards the practical implementation of $\mathrm{THz} \mathrm{BCNs}$. For example, a recent study shows that it is possible to use Perovskite to design $\mathrm{THz}$ antennas for nanoscale networks [255]. Indeed, the current research trend in the field of biomaterials will soon result in the realization of THz-based nanoscale BCNs. Since such materials could result in new types of signal sources, novel signal processing techniques are required.

\section{B. Novel Channel Models}

As discussed in Sec. III the channel model for THz in-body communications is mainly affected by absorption, spreading, and scattering. Nevertheless, the health conditions vary from person to person, due to different blood compositions, skin types, and fat concentrations that can result in distinct channel characteristics. Therefore, novel and robust channel models need to be investigated to capture these variations based on a person's health condition.

\section{Hybrid Systems}

Undoubtedly, both molecular and THz-band in-body communication paradigms can enable IBN links. However, research on molecular communication is more mature compared to the THz-based approach. Therefore, developing a hybrid molecular/THz solution can further open new opportunities. For instance, molecular communication can be used within the human body due to its noninvasive and bio-compatible nature, whereas a $\mathrm{THz}$ implanted transceiver can collect the data and transmit it to a gateway [256]. The $\mathrm{THz}$ transceiver should be able to convert the molecular signal into electrical signals and transmit it over the wireless channel to the gateway. Since there are some recent research works on optical transplants, it is also possible to use an optical transceiver to connect the inbody and on-body networks. The research on hybrid systems is still in its infancy and needs further studies.

\section{Light-weight Modulation and Coding Schemes}

As previously mentioned, the lifetime of nanodevices in IBNs is a critical system design parameter. Therefore, novel modulation and coding schemes need to be developed to account for different types of channel and noise effects. In particular, the challenge of achieving ultra-low power consumption, ultra-reliability, and low complexity needs to be addressed; the performance in terms of capacity, communication ranges, and bit error rate also needs to be studied. Generally, $\mathrm{THz}$ modulation and coding schemes are designed to maximize the channel capacity, typically in conjunction with the use of large antenna arrays to extend the communication distance [257]-[259]. However, in the case of IBNs, the priority is to reduce the overall power consumption [260]. In addition, based on the limited power budget, simple coding schemes can be 
employed to reduce coding and decoding times. This issue can be addressed by analyzing the error sources, such as the type of noise and the channel behavior, that can help examine the trade-off between decoding complexity and transmission power, leading to the design of power-efficient and low-weight channel coding schemes. In this context, researchers need to investigate low-power consumption and low-complexity modulation and coding schemes for IBNs.

\section{E. Energy Harvesting}

The devices used in $\mathrm{THz}$ BCNs are nanoscale with a limited amount of energy. Therefore, it is crucial to develop energyefficient communication paradigms and energy harvesting mechanisms for $\mathrm{THz} \mathrm{BCNs}$ to improve the network lifetime and reduce the outage probability. However, the deployment environment of BCNs is quite complicated compared to terrestrial networks. Therefore, conventional energy harvesting techniques that can collect ambient RF energy or solar power cannot be directly applied to $\mathrm{THz} \mathrm{BCNs}$ due to technological limitations [261]. Hence, researchers need to look for novel energy harvesting techniques, such as piezoelectric nanogenerators and fluidic energy harvesters for THz BCNs. Based on the harvested energy, it is also crucial to optimize the duty cycle of nanodevices to minimize the network's overall energy consumption.

\section{F. Localization}

In many applications, it is crucial to get geo-tagged sensing data from nanodevices, which is quite hard when the devices are placed in a challenging environment, such as inside the human body. In such constrained environments, conventional outdoor or indoor localization systems fail; therefore, novel solutions are required. Also, unlike the case of terrestrial networks, the size of the devices used in BCNs is in the nanoscale, which requires very accurate localization algorithms. Also, the $\mathrm{THz}$ communications range is limited, resulting in a multi-hop setup where the probability of localization error propagation is high. Nevertheless, multi-hop network localization techniques such as multidimensional scaling [262] can be investigated to estimate the location of nanodevices in BCNs.

\section{G. Big Data Analytics}

Modern smart healthcare systems use a predictive, personalized, and preventive approach that cannot be guaranteed without big data analytics. Therefore, integrating big data analytics and machine learning for THz-based nanoscale BCNs can enable various new applications, such as early detection of disease and patient-centric treatment [138]. Once $\mathrm{BCNs}$ are implemented, they generate a massive amount of data that requires the researchers to investigate various big data analytics tools. Moreover, the crucial health-related data coming from $\mathrm{BCNs}$ needs real-time analysis to take timely actions.

\section{H. Security}

Terahertz BCNs are envisioned to revolutionize the healthcare sector. However, due to the broadcasting nature of data traffic, they are vulnerable to security breaches. Recent advancements in quantum computing further increase the risk of cyber-security in BCNs. Therefore, it is crucial to secure the patient's sensitive data in THz-based BCNs from passive and active cyber attacks. For instance, recently, the authors in [263] proposed a physical layer authentication mechanism for $\mathrm{THz} \mathrm{BCNs}$, where the path loss is used as a footprint for authentication. For evaluation purposes, they used an inair $\mathrm{THz}$ system setup; however, implementing such physical layer protocols for the nano-networks in the human body is still an open research problem.

\section{Conclusions}

In this paper, we study the prospects of using the $\mathrm{THz}$ band for in-body and on-body communications, as an alternative to molecular, acoustic, optical, and radio-frequency solutions. We examine the literature on $\mathrm{THz}$ signal sources, channel and noise modeling, modulation, networking, sensing and imaging, and health and safety concerns. First, we discuss numerous $\mathrm{THz}$ signal sources for body-centric networks (BCNs), including electronic, photonic, plasmonic, and hybrid solutions. We then present the literature on channel modeling for inbody $\mathrm{THz}$ communications, where the main impediments are absorption, spreading, and scattering. Moreover, we review various types of noise sources for $\mathrm{THz}$ in-body communications. We study modulation schemes for $\mathrm{THz} \mathrm{BCNs}$, where the main idea is to design energy-efficient and lightweight techniques to reduce the overall energy consumption of the nanoscale devices. We further explain various networking protocols, including single path and multipath routing, showing that single path routing is energy efficient but unreliable, whereas multipath routing is reliable but at the cost of more power consumption. Furthermore, we highlight $\mathrm{THz}$ sensing and imaging prospects in the healthcare industry, especially in the detection of zoonotic diseases, such as Coronavirus disease. Finally, we outline several future research directions that highlight the importance of this promising research area. In a nutshell, this tutorial can be a good starting point for researchers working on $\mathrm{THz} \mathrm{BCNs}$, which still have a long way to go.

\section{REFERENCES}

[1] M. Xiao, S. Mumtaz, Y. Huang, L. Dai, Y. Li, M. Matthaiou, G. K. Karagiannidis, E. Björnson, K. Yang, C. I, and A. Ghosh, "Millimeter wave communications for future mobile networks," IEEE J. Sel. Areas Commun., vol. 35, no. 9, pp. 1909-1935, Sep. 2017.

[2] S. Rangan, T. S. Rappaport, and E. Erkip, "Millimeter-wave cellular wireless networks: Potentials and challenges," Proc. of the IEEE, vol. 102, no. 3, pp. 366-385, Mar. 2014.

[3] I. F. Akyildiz, J. M. Jornet, and C. Han, "TeraNets: Ultra-broadband communication networks in the terahertz band," IEEE Wireless Commun., vol. 21, no. 4, pp. 130-135, Aug. 2014.

[4] - "Terahertz band: Next frontier for wireless communications," Physical Commun., vol. 12, pp. 16-32, Sep. 2014.

[5] T. Kleine-Ostmann and T. Nagatsuma, "A review on terahertz communications research," J. of Infrared, Millimeter, and Terahertz Waves, vol. 32, no. 2, pp. 143-171, 2011. 
[6] Z. Chen, X. Ma, B. Zhang, Y. Zhang, Z. Niu, N. Kuang, W. Chen, L. Li, and $\mathrm{S}$. Li, "A survey on terahertz communications," China Commun., vol. 16, no. 2, pp. 1-35, Feb. 2019.

[7] K. Huang and Z. Wang, "Terahertz terabit wireless communication," IEEE Commun. Mag., vol. 12, no. 4, pp. 108-116, Jun. 2011.

[8] H. Song and T. Nagatsuma, "Present and future of terahertz communications," IEEE Trans. THz Sci. Technol., vol. 1, no. 1, pp. 256-263, Sep. 2011.

[9] N. Rajatheva, I. Atzeni, E. Bjornson, A. Bourdoux, S. Buzzi, J.-B. Dore, S. Erkucuk, M. Fuentes, K. Guan, Y. Hu et al., "White paper on broadband connectivity in 6G," arXiv preprint, 2020. [Online]. Available: https://arxiv.org/abs/2004.14247

[10] N. Rajatheva, I. Atzeni, S. Bicais, E. Bjornson, A. Bourdoux, S. Buzzi, C. D'Andrea, J.-B. Dore, S. Erkucuk, M. Fuentes et al., "Scoring the terabit/s goal: Broadband connectivity in 6G," arXiv preprint arXiv:2008.07220, 2020.

[11] A. Bourdoux, A. N. Barreto, B. van Liempd, C. de Lima, D. Dardari, D. Belot, E.-S. Lohan, G. Seco-Granados, H. Sarieddeen, H. Wymeersch et al., "6G white paper on localization and sensing," arXiv preprint arXiv:2006.01779, 2020.

[12] S. Dang, O. Amin, B. Shihada, and M.-S. Alouini, "What should 6G be?" Nature Electronics, vol. 3, no. 1, pp. 20-29, 2020.

[13] K. M. S. Huq, S. A. Busari, J. Rodriguez, V. Frascolla, W. Bazzi, and D. C. Sicker, "Terahertz-enabled wireless system for beyond-5G ultrafast networks: A brief survey," IEEE Netw., vol. 33, no. 4, pp. 89-95, Jul. 2019.

[14] J. Zhang, K. Kang, Y. Huang, M. Shafi, and A. F. Molisch, "Millimeter and $\mathrm{THz}$ wave for $5 \mathrm{G}$ and beyond," China Commun., vol. 16, no. 2, pp. iii-vi, Feb. 2019

[15] A.-A. A. Boulogeorgos, A. Alexiou, D. Kritharidis, A. Katsiotis, G. Ntouni, J. Kokkoniemi, J. Lethtomaki, M. Juntti, D. Yankova, A. Mokhtar, J.-C. Point, J. Machado, R. Elschner, C. Schubert, T. Merkle, R. Ferreira, F. Rodrigues, and J. Lima, "Wireless terahertz system architectures for networks beyond 5G," arXiv preprint, 2018. [Online]. Available: https://arxiv.org/abs/1810.12260

[16] F. Tariq, M. Khandaker, K.-K. Wong, M. Imran, M. Bennis, and M. Debbah, "A speculative study on 6G," arXiv preprint, 2019. [Online]. Available: https://arxiv.org/abs/1902.06700

[17] M. Giordani, M. Polese, M. Mezzavilla, S. Rangan, and M. Zorzi, "Toward 6G networks: Use cases and technologies," IEEE Commun. Mag., vol. 58, no. 3, pp. 55-61, 2020.

[18] C. Han, Y. Wu, Z. Chen, and X. Wang, "Terahertz communications (TeraCom): Challenges and impact on 6G wireless systems," arXiv preprint, 2019. [Online]. Available: https://arxiv.org/abs/1912.06040

[19] J.-B. Doré, D. Belot, E. Mercier, S. Bicaïs, G. Gougeon, Y. Corre, B. Miscopein, D. Kténas, and E. Strinati, "Technology roadmap for beyond 5G wireless connectivity in D-band," in 6G Summit, 2020.

[20] A. Mourad, R. Yang, P. H. Lehne, and A. De La Oliva, "A baseline roadmap for advanced wireless research beyond 5G," Electronics, vol. 9, no. 2, p. 351, 2020.

[21] W. Saad, M. Bennis, and M. Chen, "A vision of 6G wireless systems: Applications, trends, technologies, and open research problems," arXiv preprint, 2019. [Online]. Available: https://arxiv.org/abs/1902.10265

[22] H. Sarieddeen, N. Saeed, T. Y. Al-Naffouri, and M.-S. Alouini, "Next generation terahertz communications: A rendezvous of sensing, imaging, and localization," IEEE Commun. Mag., May 2020.

[23] T. Kürner and S. Priebe, "Towards $\mathrm{THz}$ communications-status in research, standardization and regulation," J. of Infrared, Millimeter, and Terahertz Waves, vol. 35, no. 1, pp. 53-62, 2014.

[24] K. Tekbıyık, A. R. Ekti, G. K. Kurt, and A. Görçin, "Terahertz band communication systems: Challenges, novelties and standardization efforts," Physical Commun., vol. 35, p. 100700, 2019.

[25] H. Elayan, O. Amin, B. Shihada, R. M. Shubair, and M. Alouini, "Terahertz band: The last piece of RF spectrum puzzle for communication systems," IEEE Open J. of the Commun. Soc., pp. 1-1, 2019.

[26] "IEEE 802.15 WPAN Terahertz Interest Group (IGthz)," http://www.ieee802.org/15/pub/IGthzOLD.html online Accessed: 14.06.2020.

[27] A. Y. Pawar, D. D. Sonawane, K. B. Erande, and D. V. Derle, "Terahertz technology and its applications," Drug Invention Today, vol. 5, no. 2, pp. $157-163,2013$

[28] G. Piro, K. Yang, G. Boggia, N. Chopra, L. A. Grieco, and A. Alomainy, "Terahertz communications in human tissues at the nanoscale for healthcare applications," IEEE Trans. Nanotechnol., vol. 14, no. 3, pp. 404-406, 2015.
[29] I. F. Akyildiz and J. M. Jornet, "Electromagnetic wireless nanosensor networks," Nano Communication Networks, vol. 1, no. 1, pp. 3 - 19, 2010.

[30] R. M. Shubair and H. Elayan, "In vivo wireless body communications: State-of-the-art and future directions," in Loughborough Antennas Propagation Conf., (LAPC), 2015, pp. 1-5.

[31] I. Akyildiz, F. Brunetti, and C. Blázquez, "Nanonetworks: A new communication paradigm," Computer Networks, vol. 52, pp. 22602279, 082008.

[32] Q. H. Abbasi, K. Yang, N. Chopra, J. M. Jornet, N. A. Abuali, K. A. Qaraqe, and A. Alomainy, "Nano-communication for biomedical applications: A review on the state-of-the-art from physical layers to novel networking concepts," IEEE Access, vol. 4, pp. 3920-3935, 2016

[33] M. Pierobon and I. F. Akyildiz, "A physical end-to-end model for molecular communication in nanonetworks," IEEE J. Sel. Areas Commun., vol. 28, no. 4, pp. 602-611, 2010.

[34] G. E. Santagati and T. Melodia, "Opto-ultrasonic communications in wireless body area nanonetworks," in Asilomar Conf. on Signals, Systems and Computers, 2013, pp. 1066-1070.

[35] T. Hogg and R. A. F. Jr.], "Acoustic communication for medical nanorobots," Nano Communication Networks, vol. 3, no. 2, pp. 83 102, 2012.

[36] Q. H. Abbasi, A. Sani, A. Alomainy, and Y. Hao, "Numerical characterization and modeling of subject-specific ultrawideband body-centric radio channels and systems for healthcare applications," IEEE Trans. Inf. Technol. Biomed., vol. 16, no. 2, pp. 221-227, 2012.

[37] Q. H. Abbasi, M. U. Rehman, K. Qaraqe, and A. Alomainy, Advances in Body-Centric Wireless Communication: Applications and State-ofthe-Art, 1st ed. Institution of Engineering and Technology, 2016.

[38] K. Yang, D. Bi, Y. Deng, R. Zhang, M. M. U. Rahman, N. A. Ali, M. A. Imran, J. M. Jornet, Q. H. Abbasi, and A. Alomainy, "A comprehensive survey on hybrid communication for internet of nano-things in context of body-centric communications," 2019. [Online]. Available: https://arxiv.org/abs/1912.09424

[39] M. Alam, N. Nurain, S. Tairin, and A. B. M. A. A. Islam, "Energyefficient transport layer protocol for hybrid communication in body area nanonetworks," in IEEE Region 10 Humanitarian Techno. Conf. (R10-HTC), 2017, pp. 674-677.

[40] K. Sayrafian-Pour, W. Yang, J. Hagedorn, J. Terrill, and K. Y. Yazdandoost, "A statistical path loss model for medical implant communication channels," in IEEE 20th Int. Symp. on Personal, Indoor and Mobile Radio Commun., 2009, pp. 2995-2999.

[41] J. Wang and Q. Wang, "Channel modeling and BER performance of an implant UWB body area link," in 2nd Int. Symp. on Applied Sciences in Biomedical and Commun. Techno., 2009, pp. 1-4.

[42] S. Støa, R. Chavez-Santiago, and I. Balasingham, "An ultra wideband communication channel model for the human abdominal region," in Proc. IEEE Global Conmun. Conf. (GLOBECOM) Workshops, 2010, pp. 246-250.

[43] K. Sayrafian, W.-B. Yang, J. Hagedorn et al., "Channel models for medical implant communication," IJWIN, vol. 17, pp. 105-112, 12 2010.

[44] D. Kurup, W. Joseph, G. Vermeeren, and L. Martens, "In-body path loss model for homogeneous human muscle, brain, fat and skin," in Proc. of the Fourth European Conf. on Antennas and Propagation, 2010, pp. 1-4.

[45] A. Khaleghi, R. Chávez-Santiago, X. Liang, I. Balasingham, V. C. M. Leung, and T. A. Ramstad, "On ultra wideband channel modeling for in-body communications," in IEEE 5th Int. Symp. on Wireless Pervasive Computing, 2010, pp. 140-145.

[46] A. Khaleghi, R. Chávez-Santiago, and I. Balasingham, "Ultra-wideband pulse-based data communications for medical implants," IET Commun., vol. 4, no. 15, pp. 1889-1897, 2010.

[47] D. Kurup, M. Scarpello, G. Vermeeren et al., "In-body path loss models for implants in heterogeneous human tissues using implantable slot dipole conformal flexible antennas," Eurasip J. on Wireless Commun. and Netw., vol. 1, 122011.

[48] A. Khaleghi, R. Chávez-Santiago, and I. Balasingham, "Ultra-wideband statistical propagation channel model for implant sensors in the human chest," IET Microwaves, Antennas Propagation, vol. 5, no. 15, pp. 1805-1812, 2011.

[49] —, "An improved ultra wideband channel model including the frequency-dependent attenuation for in-body communications," in $\mathrm{An}$ nual Int. Conf. of the IEEE Engineering in Medicine and Biology Society, 2012, pp. 1631-1634. 
[50] D. Kurup, W. Joseph, G. Vermeeren, and L. Martens, "In-body path loss model for homogeneous human tissues," IEEE Trans. Electromagn. Compat., vol. 54, no. 3, pp. 556-564, 2012.

[51] R. Chavez-Santiago, K. Sayrafian-Pour, A. Khaleghi et al., "Propagation models for IEEE 802.15.6 standardization of implant communication in body area networks," IEEE Commun. Mag., vol. 51, no. 8, pp. 80-87, 2013.

[52] R. Chavez-Santiago, C. Garcia-Pardo, A. Fornes-Leal et al., "Experimental path loss models for in-body communications within 2.36-2.5 GHz," IEEE J. Biomed. Health Inform., vol. 19, no. 3, pp. 930-937, 2015.

[53] P. A. Floor, R. Chávez-Santiago, S. Brovoll, Ø. Aardal et al., "In-body to on-body ultrawideband propagation model derived from measurements in living animals," IEEE J. Biomed. Health Inform., vol. 19 no. 3, pp. 938-948, 2015.

[54] K. M. S. Thotahewa, J. Redoutè, and M. R. Yuce, "Propagation, power absorption, and temperature analysis of UWB wireless capsule endoscopy devices operating in the human body," IEEE Trans. Microw. Theory Techn., vol. 63, no. 11, pp. 3823-3833, 2015.

[55] C. Garcia-Pardo, A. Fornes-Leal, N. Cardona et al., "Experimental ultra wideband path loss models for implant communications," in IEEE 27th Annual Int. Symp. on Personal, Indoor, and Mobile Radio Commun., (PIMRC), 2016, pp. 1-6.

[56] Y. Shimizu, T. Furukawa, D. Anzai, and J. Wang, "Performance improvement by transmit diversity technique for implant ultra-wideband communication," IET Microwaves, Antennas Propagation, vol. 10, no. 10, pp. 1106-1112, 2016.

[57] I. T. Javed and I. H. Naqvi, "Frequency band selection and channel modeling for WNSN applications using simplenano," in IEEE Int. Conf. on Commun., (ICC), 2013, pp. 5732-5736.

[58] H. Elayan, R. M. Shubair, J. M. Jornet, and P. Johari, "Terahertz channel model and link budget analysis for intrabody nanoscale communication," IEEE Trans. Nanobiosci., vol. 16, no. 6, pp. 491-503, 2017.

[59] K. Yang, A. Pellegrini, M. O. Munoz, A. Brizzi, A. Alomainy, and Y. Hao, "Numerical analysis and characterization of $\mathrm{THz}$ propagation channel for body-centric nano-communications," IEEE Trans. THz Sci. Technol., vol. 5, no. 3, pp. 419-426, 2015.

[60] Q. H. Abbasi, H. El Sallabi, N. Chopra, K. Yang, K. A. Qaraqe, and A. Alomainy, "Terahertz channel characterization inside the human skin for nano-scale body-centric networks," IEEE Trans. THz Sci. Technol., vol. 6, no. 3, pp. 427-434, 2016.

[61] C. B. Reid, G. Reese, A. P. Gibson, and V. P. Wallace, "Terahertz time-domain spectroscopy of human blood," IEEE J. Biomed. Health Inform., vol. 17, no. 4, pp. 774-778, 2013.

[62] N. Chopra, K. Yang, J. Upton, A. Alomainy, M. Philpott, and Y. Hao, "Understanding and characterizing nanonetworks for healthcare monitoring applications," in IEEE MTT-S Int. Microwave Workshop Series on RF and Wireless Technologies for Biomedical and Healthcare Applications (IMWS-Bio2014), 2014, pp. 1-3.

[63] K. Yang, N. Chopra, J. Upton, Y. Hao, M. Philpott, A. Alomainy, Q. H. Abbasi, and K. Qaraqe, "Characterising skin-based nano-networks for healthcare monitoring applications at THz," in IEEE Int. Symposium on Antennas and Propagation USNC/URSI National Radio Science Meeting, 2015, pp. 199-200.

[64] K. I. Zaytsev, A. A. Gavdush, N. V. Chernomyrdin, and S. O. Yurchenko, "Highly accurate in vivo terahertz spectroscopy of healthy skin: Variation of refractive index and absorption coefficient along the human body," IEEE Trans. THz Sci. Technol., vol. 5, no. 5, pp. 817827, 2015.

[65] N. Chopra, K. Yang, Q. H. Abbasi, K. A. Qaraqe, M. Philpott, and A. Alomainy, "THz time-domain spectroscopy of human skin tissue for in-body nanonetworks," IEEE Trans. THz Sci. Technol., vol. 6, no. 6, pp. 803-809, 2016.

[66] H. Elayan, R. M. Shubair, J. M. Jornet, and R. Mittra, "Multilayer intrabody terahertz wave propagation model for nanobiosensing applications," Nano Commun. Netw., vol. 14, pp. 9 - 15, 2017.

[67] S. E. Trevlakis, A.-A. A. Boulogeorgos, N. D. Chatzidiamantis, and G. K. Karagiannidis, "All-optical cochlear implants," 2020. [Online]. Available: https://arxiv.org/abs/2006.11577

[68] A. Julien-Vergonjanne, S. Sahuguède, and L. Chevalier, Optical Wireless Body Area Networks for Healthcare Applications. Cham: Springer International Publishing, 2016, pp. 569-587.

[69] A. Sangwan, H. Pandey, P. Johari, and J. M. Jornet, "Increasing the communication distance between nano-biosensing implants and wearable devices," in SPAWC, Jun. 2018, pp. 1-5.
[70] A.-A. A. Boulogeorgos, S. E. Trevlakis, and N. D. Chatzidiamantis, "Optical wireless communications for in-body and transdermal biomedical applications," 2020. [Online]. Available: https://arxiv.org/ abs/2005.00405

[71] O. Haddad, M. Khalighi, S. Zvanovec, and M. Adel, "Channel characterization and modeling for optical wireless body-area networks," IEEE Open J. of the Commun. Soc., vol. 1, pp. 760-776, 2020.

[72] M. Ş. Kuran, H. B. Yilmaz, T. Tugcu, and B. Özerman, "Energy model for communication via diffusion in nanonetworks," Nano Commun. Netw., vol. 1, no. 2, pp. $86-95,2010$.

[73] M. Gregori, I. Llatser, A. Cabellos-Aparicio, and E. Alarcón, "Physical channel characterization for medium-range nanonetworks using flagellated bacteria," Computer Netw., vol. 55, no. 3, pp. 779 - 791, 2011.

[74] Y. Chahibi, M. Pierobon, S. O. Song, and I. F. Akyildiz, "A molecular communication system model for particulate drug delivery systems," IEEE Trans. Biomed. Eng., vol. 60, no. 12, pp. 3468-3483, 2013.

[75] L. Galluccio, S. Palazzo, and G. E. Santagati, "Characterization of molecular communications among implantable biomedical neuroinspired nanodevices," Nano Commun. Netw., vol. 4, no. 2, pp. 53 64, 2013.

[76] P. He, Y. Mao, Q. Liu, P. Liò, and K. Yang, "Channel modelling of molecular communications across blood vessels and nerves," in Proc. IEEE Int. Conf. Commun. (ICC), 2016, pp. 1-6.

[77] A. Al-Helali, B. Liang, and N. Nasser, "Novel molecular signaling method and system for molecular communication in human body," IEEE Access, vol. 8, pp. 119361-119375, 2020.

[78] I. F. Akyildiz, M. Ghovanloo, U. Guler, T. Ozkaya-Ahmadov, A. F. Sarioglu, and B. D. Unluturk, "PANACEA: An internet of bio-nanothings application for early detection and mitigation of infectious diseases," IEEE Access, vol. 8, pp. 140 512-140 523, 2020.

[79] G. E. Santagati and T. Melodia, "Sonar inside your body: Prototyping ultrasonic intra-body sensor networks," in IEEE Conf. on Computer Commun., 2014, pp. 2679-2687.

[80] G. E. Santagati, T. Melodia, L. Galluccio, and S. Palazzo, "Medium access control and rate adaptation for ultrasonic intrabody sensor networks," IEEE/ACM Trans. Netw., vol. 23, no. 4, pp. 1121-1134, 2015.

[81] D. Anzai, K. Katsu, R. Chavez-Santiago et al., "Experimental evaluation of implant UWB-IR transmission with living animal for body area networks," IEEE Trans. Microw. Theory Techn., vol. 62, no. 1, pp. 183-192, 2014.

[82] J. Kokkoniemi, J. Lehtomäki, and M. Juntti, "A discussion on molecular absorption noise in the terahertz band," Nano Commun. Netw., vol. 8 , pp. $35-45,2016$.

[83] Ke Yang, Yang Hao, A. Alomainy, Q. H. Abbasi, and K. Qaraqe, "Channel modelling of human tissues at terahertz band," in IEEE Wireless Commun. and Netw. Conf. Works., (WCNCW), 2016, pp. 218 221.

[84] R. Zhang, K. Yang, A. Alomainy, Q. H. Abbasi, K. Qaraqe, and R. M. Shubair, "Modelling of the terahertz communication channel for invivo nano-networks in the presence of noise," in 16th Mediterranean Microwave Symposium (MMS), 2016, pp. 1-4.

[85] H. Elayan, P. Johari, R. M. Shubair, and J. M. Jornet, "Photothermal modeling and analysis of intrabody terahertz nanoscale communication," IEEE Trans. Nanobiosci., vol. 16, no. 8, pp. 755-763, 2017.

[86] R. Zhang, K. Yang, Q. H. Abbasi, K. A. Qaraqe, and A. Alomainy, "Analytical modelling of the effect of noise on the terahertz invivo communication channel for body-centric nano-networks," Nano Commun. Netw., vol. 15, pp. 59 - 68, 2018.

[87] H. Elayan, C. Stefanini, R. M. Shubair, and J. M. Jornet, "End-to-end noise model for intra-body terahertz nanoscale communication," IEEE Trans. Nanobiosci., vol. 17, no. 4, pp. 464-473, 2018.

[88] M. Pierobon and I. F. Akyildiz, "Diffusion-based noise analysis for molecular communication in nanonetworks," IEEE Trans. Signal Process., vol. 59, no. 6, pp. 2532-2547, 2011.

[89] — "Noise analysis in ligand-binding reception for molecular communication in nanonetworks," IEEE Trans. Signal Process., vol. 59, no. 9, pp. 4168-4182, 2011.

[90] K. V. Srinivas, A. W. Eckford, and R. S. Adve, "Molecular communication in fluid media: The additive inverse gaussian noise channel," IEEE Trans. Inf. Theory, vol. 58, no. 7, pp. 4678-4692, 2012.

[91] Y. Chahibi and I. F. Akyildiz, "Molecular communication noise and capacity analysis for particulate drug delivery systems," IEEE Trans. Commun., vol. 62, no. 11, pp. 3891-3903, 2014.

[92] Y. Shimizu, D. Anzai, R. Chavez-Santiago, P. A. Floor, I. Balasingham, and J. Wang, "Performance evaluation of an ultra-wideband transmit 
diversity in a living animal experiment," IEEE Trans. Microw. Theory Techn., vol. 65, no. 7, pp. 2596-2606, 2017.

[93] E. Miralles, C. Andreu, M. Cabedo-Fabrés, M. Ferrando-Bataller, and J. F. Monserrat, "UWB on-body slotted patch antennas for inbody communications," in 11th European Conf. on Antennas and Propagation (EUCAP), 2017, pp. 167-171.

[94] G. Piro, P. Bia, G. Boggia, D. Caratelli, L. Grieco, and L. Mescia, "Terahertz electromagnetic field propagation in human tissues: A study on communication capabilities," Nano Commun. Netw., vol. 10, pp. 51 $-59,2016$

[95] D. Arifler, "Capacity analysis of a diffusion-based short-range molecular nano-communication channel," Computer Networks, vol. 55, no. 6 pp. $1426-1434,2011$.

[96] M. Şükrü Kuran, H. B. Yilmaz, T. Tugcu, and I. F. Akyildiz, "Interference effects on modulation techniques in diffusion based nanonetworks," Nano Commun. Netw., vol. 3, no. 1, pp. 65 - 73, 2012.

[97] M. Pierobon and I. F. Akyildiz, "Capacity of a diffusion-based molecular communication system with channel memory and molecular noise," IEEE Trans. Inf. Theory, vol. 59, no. 2, pp. 942-954, 2013.

[98] Y. Chahibi, M. Pierobon, and S. O. Song, "Molecular communication model of nanoparticle-body interactions in particulate drug delivery systems," in Asilomar Conf. on Signals, Systems and Computers, 2013, pp. 1051-1055.

[99] R. Chávez-Santiago, I. Balasingham, J. Bergsland et al., "Experimental implant communication of high data rate video using an ultra wideband radio link," in 35th Annual Int. Conf. of the IEEE Engineering in Medicine and Biology Society (EMBC), 2013, pp. 5175-5178.

[100] K. Yang, Q. H. Abbasi, K. Qaraqe, A. Alomainy, and Y. Hao, "Bodycentric nano-networks: EM channel characterisation in water at the terahertz band," in Asia-Pacific Microwave Conference, 2014, pp. 531533.

[101] N. Garralda, I. Llatser, A. Cabellos-Aparicio et al., "Diffusion-based physical channel identification in molecular nanonetworks," Nano Commun. Netw., vol. 2, no. 4, pp. 196 - 204, 2011.

[102] Y. Chahibi and I. Balasingham, "An intra-body molecular communication networks framework for continuous health monitoring and diagnosis," in 37th Annual Int. Conf. of the IEEE Engineering in Medicine and Biology Society (EMBC), 08 2015, pp. 4077-4080.

[103] J. M. Jornet and I. F. Akyildiz, "Femtosecond-long pulse-based modulation for terahertz band communication in nanonetworks," IEEE Trans. Commun., vol. 62, no. 5, pp. 1742-1754, May. 2014.

[104] A. K. Vavouris, F. D. Dervisi, V. K. Papanikolaou, and G. K. Karagiannidis, "An energy efficient modulation scheme for body-centric nano-communications in the THz band," in 7th Int. Conf. on Modern Circuits and Sys. Techno., (MOCAST), 2018, pp. 1-4.

[105] R. Zhang, K. Yang, Q. H. Abbasi, K. A. Qaraqe, and A. Alomainy, "Analytical characterisation of the terahertz in-vivo nano-network in the presence of interference based on TS-OOK communication scheme," IEEE Access, vol. 5, pp. $10172-10$ 181, 2017.

[106] D. R. Dhatchayeny, W. A. Cahyadi, S. R. Teli, and Y. Chung, "A novel optical body area network for transmission of multiple patient vital signs," in Ninth Int. Conf. on Ubiquitous and Future Networks (ICUFN), 2017, pp. 542-544.

[107] M. S. Kuran, H. B. Yilmaz, T. Tugcu, and I. F. Akyildiz, "Modulation techniques for communication via diffusion in nanonetworks," in Proc. IEEE Int. Conf. Commun. (ICC), 2011, pp. 1-5.

[108] I. Llatser, A. Cabellos-Aparicio, M. Pierobon, and E. Alarcon, "Detection techniques for diffusion-based molecular communication," IEEE J. Sel. Areas Commun., vol. 31, no. 12, pp. 726-734, 2013.

[109] H. Arjmandi, A. Gohari, M. N. Kenari, and F. Bateni, "Diffusionbased nanonetworking: A new modulation technique and performance analysis," IEEE Commun. Lett., vol. 17, no. 4, pp. 645-648, 2013.

[110] P. Akhkandi, A. Keshavarz-Haddad, and A. Jamshidi, "A new channel code for decreasing inter-symbol-interference in diffusion based molecular communications," in 8th Int. Symp. on Telecommun., (IST), 2016, pp. 277-281.

[111] Y. Lu, X. Wang, M. D. Higgins, A. Noel, N. Neophytou, and M. S. Leeson, "Energy requirements of error correction codes in diffusion-based molecular communication systems," Nano Commun. Netw., vol. 11, pp. $24-35,2017$

[112] A. Zare and A. Jamshidi, "Receiver design and performance analysis for pulse position modulation technique in diffusion-based molecular communication," Nano Commun. Netw., vol. 21, p. 100256, 2019.

[113] E. Demirors, G. Alba, G. E. Santagati, and T. Melodia, "High data rate ultrasonic communications for wireless intra-body networks," in IEEE Int. Symp. on Local and Metropolitan Area Networks (LANMAN), 2016, pp. $1-6$.
[114] Wangjong Lee, Seung Hyong Rhee, Youjin Kim, and Hyungsoo Lee, "An efficient multi-channel management protocol for wireless body area networks," in Int. Conf. on Information Networking, 2009, pp. $1-5$.

[115] M. Pierobon, J. M. Jornet, N. Akkari, and S. A. I. F. Akyildiz, "A routing framework for energy harvesting wireless nanosensor networks in the terahertz band," Wireless Netw., vol. 20, p. 1169-1183, 2014.

[116] S. Canovas-Carrasco, A.-J. Garcia-Sanchez, and J. Garcia-Haro, "A nanoscale communication network scheme and energy model for a human hand scenario," Nano Commun. Netw., vol. 15, pp. 17 - 27, 2018.

[117] L. Feng, Q. Yang, D. Park, and K. S. Kwak, "Energy efficient nano-node association and resource allocation for hierarchical nanocommunication networks," IEEE Trans. Mol. Biol. Multi-Scale Commun., vol. 4, no. 4, pp. 208-220, 2018.

[118] G. Ardeshiri, A. Jamshidi, and A. Keshavarz-Haddad, "Performance analysis of decode and forward relay network in diffusion based molecular communication," in Iranian Conf. on Electrical Engineering (ICEE), 2017, pp. 1992-1997.

[119] S. Basagni, M. Conti, S. Giordano, and I. Stojmenovic, Advances in Underwater Acoustic Networking. Wiley-IEEE Press, 2013, pp. 804852.

[120] F. Dressler and F. Kargl, "Towards security in nano-communication: Challenges and opportunities," Nano Commun. Netw., vol. 3, no. 3, pp. $151-160,2012$.

[121] M. Usman, M. R. Asghar, I. S. Ansari, and M. Qaraqe, "Security in wireless body area networks: From in-body to off-body communications," IEEE Access, vol. 6, pp. 58 064-58 074, 2018.

[122] S. M. Riazul Islam, F. Ali, H. Moon, and K. Kwak, "Secure channel for molecular communications," in Int. Conf. on Info. and Commun. Techno. Conv., (ICTC), 2017, pp. 1-4.

[123] L. Mucchi, A. Martinelli, S. Jayousi, S. Caputo, and M. Pierobon, "Secrecy capacity and secure distance for diffusion-based molecular communication systems," IEEE Access, vol. 7, pp. 110687-110697, 2019.

[124] T. Wu, L. Tan, Q. Shao et al., "Chinese adult anatomical models and the application in evaluation of wideband RF EMF exposure," Physics in medicine and biology, vol. 56, pp. 2075-89, 032011.

[125] S. Castelló-Palacios, C. Garcia-Pardo, A. Fornes-Leal, N. Cardona, and A. Vallés-Lluch, "Tailor-made tissue phantoms based on acetonitrile solutions for microwave applications up to $18 \mathrm{GHz}$," IEEE Trans. Microw. Theory Techn., vol. 64, no. 11, pp. 3987-3994, 2016.

[126] _ - "Wideband phantoms of different body tissues for heterogeneous models in body area networks," in 39th Annual Int. Conf. of the IEEE Engineering in Medicine and Biology Society (EMBC), 2017, pp. 30323035 .

[127] N. Farsad, D. Pan, and A. Goldsmith, "A novel experimental platform for in-vessel multi-chemical molecular communications," in IEEE Global Commun. Conf., (GLOBECOM), 2017, pp. 1-6.

[128] H. Unterweger, J. Kirchner, W. Wicke et al., "Experimental molecular communication testbed based on magnetic nanoparticles in duct flow," in IEEE 19th Int. Works. on Signal Process. Adv. in Wireless Commun., (SPAWC), 2018, pp. 1-5.

[129] J. M. Jornet and I. F. Akyildiz, "Channel modeling and capacity analysis for electromagnetic wireless nanonetworks in the terahertz band," IEEE Trans. Wireless Commun., vol. 10, no. 10, pp. 3211-3221, 2011.

[130] M. R. da Costa, O. Kibis, and M. Portnoi, "Carbon nanotubes as a basis for terahertz emitters and detectors," Microelectronics J., vol. 40, no. 4 , pp. $776-778,2009$.

[131] A. Nikpaik, A. H. M. Shirazi, A. Nabavi, S. Mirabbasi, and S. Shekhar, "A 219-to-231 GHz frequency-multiplier-based VCO with 3\% peak DC-to-RF efficiency in 65-nm CMOS," IEEE J. Solid-State Circuits, vol. 53, no. 2, pp. 389-403, Feb. 2018

[132] H. Aghasi, A. Cathelin, and E. Afshari, "A 0.92-THz SiGe power radiator based on a nonlinear theory for harmonic generation," IEEE J. Solid-State Circuits, vol. 52, no. 2, pp. 406-422, Feb. 2017.

[133] J. M. Jornet and I. F. Akyildiz, "Graphene-based nano-antennas for electromagnetic nanocommunications in the terahertz band," in Proc. of the Fourth European Conf. on Antennas and Propagation, 2010, pp. $1-5$.

[134] Y. Nishi, Scaling Limits of Silicon CMOS and NonSilicon Opportunities. Wiley-IEEE Press, 01 2007, pp. 203-211.

[135] S. Ghafoor, N. Boujnah, M. H. Rehmani, and A. Davy, "MAC protocols for terahertz communication: A comprehensive survey," arXiv preprint, 2019. [Online]. Available: https://arxiv.org/abs/1904.11441 
[136] I. F. Akyildiz and J. M. Jornet, "The internet of nano-things," IEEE Wireless Commun., vol. 17, no. 6, pp. 58-63, 2010.

[137] J. M. Jornet and I. F. Akyildiz, "The internet of multimedia nanothings," Nano Commun. Netw., vol. 3, no. 4, pp. 242 - 251, 2012

[138] A. Rizwan, A. Zoha, R. Zhang, W. Ahmad, K. Arshad, N. Abu Ali, A. Alomainy, M. A. Imran, and Q. H. Abbasi, "A review on the role of nano-communication in future healthcare systems: A big data analytics perspective," IEEE Access, vol. 6, pp. 41 903-41 920, 2018.

[139] S. Balasubramaniam and J. Kangasharju, "Realizing the internet of nano things: Challenges, solutions, and applications," Computer, vol. 46, no. 2, pp. 62-68, 2013.

[140] I. F. Akyildiz, M. Pierobon, S. Balasubramaniam, and Y. Koucheryavy, "The internet of bio-nano things," IEEE Commun. Mag., vol. 53, no. 3, pp. 32-40, 2015.

[141] M. H. Miraz, M. Ali, P. S. Excell, and R. Picking, "A review on internet of things (IoT), internet of everything (IoE) and internet of nano things (IoNT)," in Internet Techno. and App., (ITA), 2015, pp. 219-224.

[142] F. Dressler and S. Fischer, "Connecting in-body nano communication with body area networks: Challenges and opportunities of the internet of nano things," Nano Commun. Netw., vol. 6, no. 2, pp. 29 - 38, 2015.

[143] F. Lemic, R. U. Akbar, J. Marquez-Barja, and J. Famaey, "Assessing the reliability of energy harvesting terahertz nanonetworks for controlling software-defined metamaterials," in Proc. of the Sixth Annual ACM Int. Conf. on Nanoscale Comput. and Commun., 2019, pp. 1-6.

[144] N. Rikhtegar, "A brief survey on molecular and electromagnetic communications in nano-networks," Int. J. of Computer App., vol. 79, no. 3, pp. 16-28, 2013.

[145] V. Petrov, D. Moltchanov, and Y. Koucheryavy, "Applicability assessment of terahertz information showers for next-generation wireless networks," in Proc. IEEE Int. Conf. Commun. (ICC), May. 2016, pp. $1-7$.

[146] F. Lemic, S. Abadal, W. Tavernier, P. Stroobant, D. Colle, E. Alarcón, J. Marquez-Barja, and J. Famaey, "Survey on terahertz nanocommunication and networking: A top-down perspective," arXiv preprint, 2019. [Online]. Available: https://arxiv.org/abs/1909.05703

[147] P. Hillger, J. Grzyb, R. Jain, and U. R. Pfeiffer, "Terahertz imaging and sensing applications with silicon-based technologies," IEEE Trans. THz Sci. Technol., vol. 9, no. 1, pp. 1-19, 2019.

[148] R. Han, Z. Hu, C. Wang, J. Holloway, X. Yi, M. Kim, and J. Mawdsley, "Filling the gap: Silicon terahertz integrated circuits offer our best bet," IEEE Microw. Mag., vol. 20, no. 4, pp. 80-93, 2019.

[149] D. M. Mittleman, "Perspective: Terahertz science and technology," Journal of Applied Physics, vol. 122, no. 23, p. 230901, 2017

[150] B. Heinemann, H. Rücker, R. Barth et al., "SiGe HBT with fx/fmax of $505 \mathrm{GHz} / 720 \mathrm{GHz}$," in IEEE Int. Electron Devices Meeting (IEDM), 2016, pp. 3.1.1-3.1.4.

[151] M. Urteaga, Z. Griffith, M. Seo, J. Hacker, and M. J. W. Rodwell, "InP HBT technologies for THz integrated circuits," Proc. of the IEEE, vol. 105, no. 6, pp. 1051-1067, Jun. 2017.

[152] C. R. Bolognesi, R. Flückiger, M. Alexandrova, W. Quan, R. Lövblom, and O. Ostinelli, "InP/GaAsSb DHBTs for $\mathrm{THz}$ applications and improved extraction of their cutoff frequencies," in IEEE Int. Electron devices meeting (IEDM), 2016, pp. 29-5.

[153] K. Sengupta, "Integrated circuits for terahertz communication beyond 100 GHz: Are we there yet?" in Proc. IEEE Int. Conf. Commun. (ICC), May. 2019, pp. 1-6.

[154] W. R. Deal, K. Leong, A. Zamora, B. Gorospe, K. Nguyen, and X. B Mei, "A $660 \mathrm{GHz}$ up-converter for THz communications," in IEEE Compound Semicond. Integr. Circuit Symp. (CSICS), Oct. 2017, pp. $1-4$.

[155] A. Leuther, A. Tessmann, P. Doria et al., "20 nm metamorphic HEMT technology for terahertz monolithic integrated circuits," in 2014 9th European Microw. Integr. Circuit Conf., Oct. 2014, pp. 84-87.

[156] X. Mei, W. Yoshida, M. Lange, J. Lee et al., "First demonstration of amplification at $1 \mathrm{THz}$ Using 25-nm InP high electron mobility transistor process," IEEE Electron Device Lett., vol. 36, no. 4, pp. 327-329, 2015

[157] I. Mehdi, J. V. Siles, C. Lee, and E. Schlecht, "THz diode technology: Status, prospects, and applications," Proc. of the IEEE, vol. 105, no. 6, pp. 990-1007, Jun. 2017.

[158] F. Ahmed, M. Furqan, B. Heinemann, and A. Stelzer, " $0.3-\mathrm{THz}$ SiGe-based high-efficiency push-push VCOs with $>1$-mW peak output power employing common-mode impedance enhancement," IEEE Trans. Microw. Theory Techn., vol. 66, no. 3, pp. 1384-1398, 2018.

[159] P. Hillger, J. Grzyb, S. Malz, B. Heinemann, and U. Pfeiffer, "A lensintegrated $430 \mathrm{GHz}$ SiGe HBT source with up to $-6.3 \mathrm{dBm}$ radiated power," in IEEE Radio Frequency Integrated Circuits Symp., (RFIC), 2017, pp. 160-163.

[160] R. Han, C. Jiang, A. Mostajeran, M. Emadi et al., "A SiGe terahertz heterodyne imaging transmitter with $3.3 \mathrm{~mW}$ radiated power and fullyintegrated phase-locked loop," IEEE J. Solid-State Circuits, vol. 50, no. 12 , pp. 2935-2947, 2015.

[161] Z. Hu, M. Kaynak, and R. Han, "High-power radiation at $1 \mathrm{THz}$ in silicon: A fully scalable array using a multi-functional radiating mesh structure," IEEE J. Solid-State Circuits, vol. 53, no. 5, pp. 1313-1327, 2018.

[162] T. Nagatsuma, G. Ducournau, and C. C. Renaud, "Advances in terahertz communications accelerated by photonics," Nature Photonics, vol. 10 no. 6 , p. 371,2016

[163] Q. Lu, D. Wu, S. Sengupta, S. Slivken, and M. Razeghi, "Room temperature continuous wave, monolithic tunable $\mathrm{THz}$ sources based on highly efficient mid-infrared quantum cascade lasers," Scientific reports, vol. 6, p. 23595, 2016.

[164] S.-W. Huang, J. Yang, S.-H. Yang et al., "Globally stable microresonator turing pattern formation for coherent high-power $\mathrm{THz}$ radiation on-chip," Physical Review X, vol. 7, no. 4, p. 041002, 2017.

[165] H. Song, K. Ajito, Y. Muramoto, A. Wakatsuki, T. Nagatsuma, and N. Kukutsu, "Uni-travelling-carrier photodiode module generating 300 GHz power greater than $1 \mathrm{~mW}$," IEEE Microw. Wireless Compon. Lett., vol. 22, no. 7, pp. 363-365, Jul. 2012.

[166] M. Feiginov, C. Sydlo, O. Cojocari, and P. Meissner, "Resonanttunnelling-diode oscillators operating at frequencies above $1.1 \mathrm{THz}$," Applied Physics Letters, vol. 99, no. 23, p. 233506, 2011.

[167] Y. Koyama, R. Sekiguchi, and T. Ouchi, "Oscillations up to $1.40 \mathrm{THz}$ from resonant-tunneling-diode-based oscillators with integrated patch antennas," Applied Physics Express, vol. 6, no. 6, p. 064102, Jun. 2013.

[168] T. Maekawa, H. Kanaya, S. Suzuki, and M. Asada, "Oscillation up to $1.92 \mathrm{THz}$ in resonant tunneling diode by reduced conduction loss," Applied Physics Express, vol. 9, no. 2, pp. 04-10, Jan. 2016.

[169] R. Izumi, S. Suzuki, and M. Asada, "1.98 THz resonant-tunnelingdiode oscillator with reduced conduction loss by thick antenna electrode," in 42nd Int. Conf. on Infrared, Millimeter, and Terahertz. Waves (IRMMW-THz), 2017, pp. 1-2.

[170] K. Sengupta, T. Nagatsuma, and D. M. Mittleman, "Terahertz integrated electronic and hybrid electronic-photonic systems," Nature Electronics, vol. 1, no. 12, p. 622, 2018.

[171] K. K. O, W. Choi, Q. Zhong, N. Sharma, Y. Zhang, R. Han, Z. Ahmad, D. Kim, S. Kshattry, I. R. Medvedev, D. J. Lary, H. Nam, P. Raskin, and I. Kim, "Opening terahertz for everyday applications," IEEE Commun. Mag., vol. 57, no. 8, pp. 70-76, Aug. 2019.

[172] S. Koenig, D. Lopez-Diaz, J. Antes, F. Boes, R. Henneberger, A. Leuther, A. Tessmann, R. Schmogrow, D. Hillerkuss, R. Palmer et al., "Wireless sub-THz communication system with high data rate," Nature photonics, vol. 7, no. 12, p. 977, 2013.

[173] H. Hafez, S. Kovalev, J. Deinert, Z. Mics, B. Green et al., "Extremely efficient terahertz high-harmonic generation in graphene by hot Dirac fermions." Nature, 2018.

[174] L. Ju, B. Geng, J. Horng et al., "Graphene plasmonics for tunable terahertz metamaterials," Nature Nanotechno., vol. 6, no. 10, p. 630 , 2011.

[175] K. S. Novoselov, V. Fal, L. Colombo, P. Gellert, M. Schwab, K. Kim et al., "A roadmap for graphene," Nature, vol. 490, no. 7419, p. 192 , 2012.

[176] A. C. Ferrari, F. Bonaccorso, V. Fal'Ko et al., "Science and technology roadmap for graphene, related two-dimensional crystals, and hybrid systems," Nanoscale, vol. 7, no. 11, pp. 4598-4810, 2015.

[177] Z. Xu, X. Dong, and J. Bornemann, "Design of a reconfigurable MIMO system for THz communications based on graphene antennas," IEEE Trans. THz Sci. Technol., vol. 4, no. 5, pp. 609-617, Sep. 2014

[178] G. W. Hanson, "Dyadic green's functions for an anisotropic, non-local model of biased graphene," IEEE Trans. Antennas Propag., vol. 56, no. 3, pp. 747-757, 2008

[179] J. M. Jornet and I. F. Akyildiz, "Graphene-based plasmonic nanoantenna for terahertz band communication in nanonetworks," IEEE $J$. Sel. Areas Commun., vol. 31, no. 12, pp. 685-694, Dec. 2013.

[180] N. Thawdar, M. Andrello III, and J. M. Jornet, "Modeling and performance analysis of a reconfigurable plasmonic nano-antenna array architecture for terahertz communications," in Proc. of the 5th ACM Int. Conf. on Nanoscale Comput. and Commun., 2018, pp. 20-26.

[181] G. J. Wilmink and J. E. Grundt, "Invited review article: Current state of research on biological effects of terahertz radiation," J. of Infrared, Millimeter, and Terahertz Waves, vol. 32, no. 10, pp. 1074-1122, 2011. 
[182] J. M. Jornet and I. F. Akyildiz, "Channel capacity of electromagnetic nanonetworks in the terahertz band," in Proc. IEEE Int. Conf. Commun. (ICC), May. 2010, pp. 1-6.

[183] K. Yang, A. Alomainy, and Y. Hao, "In-vivo characterisation and numerical analysis of the $\mathrm{THz}$ radio channel for nanoscale body-centric wireless networks," in 2013 USNC-URSI Radio Science Meeting (Joint with AP-S Symposium), 2013, pp. 218-219.

[184] H. Elayan, R. M. Shubair, A. Alomainy, and K. Yang, "In-vivo terahertz EM channel characterization for nano-communications in WBANs," in IEEE Int. Symp. on Antennas and Propagation (APSURSI), 2016, pp. 979-980.

[185] J. T. Kindt and C. A. Schmuttenmaer, "Far-infrared dielectric properties of polar liquids probed by femtosecond terahertz pulse spectroscopy," The J. of Physical Chemistry, vol. 100, no. 24, pp. 10373-10379, 1996.

[186] M. Youssef, F. Ghanim, N. Imad, A. Alqasim, and R. Shubair, "Design of intra-body nano-communication network for future nano-medicine," 2018. [Online]. Available: https://arxiv.org/abs/1810.00186

[187] F. Box, "Utilization of atmospheric transmission losses for interferenceresistant communications," IEEE Trans. Commun., vol. 34, no. 10, pp. 1009-1015, 1986.

[188] A. N. Pal and A. Ghosh, "Ultralow noise field-effect transistor from multilayer graphene," Applied Physics Letters, vol. 95, no. 8, p. 082105, 2009.

[189] Z. L. Wang, "Towards self-powered nanosystems: From nanogenerators to nanopiezotronics," Advanced Functional Materials, vol. 18, no. 22, pp. 3553-3567, 2008 .

[190] Q. Xia and J. M. Jornet, "Routing protocol design for directional and buffer-limited terahertz communication networks," in IEEE Int. Conf. on Commun., (ICC), 2020, pp. 1-7.

[191] G. Piro, L. A. Grieco, G. Boggia, and P. Camarda, "Nano-Sim: Simulating electromagnetic-based nanonetworks in the Network Simulator 3," in Workshop on NS-3, Jul. 2013.

[192] F. Afsana, M. Asif-Ur-Rahman, M. R. Ahmed, M. Mahmud, and M. S. Kaiser, "An energy conserving routing scheme for wireless body sensor nanonetwork communication," IEEE Access, vol. 6, pp. 9186-9200, 2018.

[193] J. Xu, R. Zhang, and Z. Wang, "An energy efficient multi-hop routing protocol for terahertz wireless nanosensor networks," in Wireless Algorithms, Systems, and Applications, 2016, pp. 367-376.

[194] H. Yu, B. Ng, W. K. G. Seah, and Y. Qu, "TTL-based efficient forwarding for the backhaul tier in nanonetworks," in 14th IEEE Annual Consumer Commun. Netw. Conf., (CCNC), 2017, pp. 554-559.

[195] A. Galal and X. Hesselbach, "Probability-based path discovery protocol for electromagnetic nano-networks," Computer Networks, vol. 174, p. 107246, 2020.

[196] S. R. Neupane, "Routing in resource constrained sensor nanonetworks," Master's thesis, Tampere University of Technology, 2014.

[197] A. Tsioliaridou, C. Liaskos, S. Ioannidis, and A. Pitsillides, "CORONA A coordinate and routing system for nanonetworks," in Proc. of the Second Annual Int. Conf. on Nanoscale Comput. and Commun., 2015, pp. $1-6$.

[198] A. Tsioliaridou, C. Liaskos, L. Pachis, S. Ioannidis, and A. Pitsillides, "N3: Addressing and routing in 3D nanonetworks," in 23rd Int. Conf. on Telecommun., (ICT), 2016, pp. 1-6.

[199] A. Tsioliaridou, C. Liaskos, E. Dedu, and S. Ioannidis, "Packet routing in 3D nanonetworks: A lightweight, linear-path scheme," Nano Commun. Netw., vol. 12, pp. 63 - 71, 2017.

[200] A. Tsioliaridou, C. Liaskos, S. Ioannidis, and A. Pitsillides, "Lightweight, self-tuning data dissemination for dense nanonetworks," Nano Communication Networks, vol. 8, pp. 2 - 15, 2016.

[201] J. Misra and D. Gries, "Finding repeated elements," Science of Computer Programming, vol. 2, no. 2, pp. 143 - 152, 1982.

[202] C. Liaskos, A. Tsioliaridou, S. Ioannidis, N. Kantartzis, and A. Pitsillides, "A deployable routing system for nanonetworks," in Proc. IEEE Int. Conf. Commun. (ICC), 2016, pp. 1-6.

[203] S. J. Park, S. H. Cha, G. A. Shin, and Y. H. Ahn, "Sensing viruses using terahertz nano-gap metamaterials," Biomed. Opt. Express, vol. 8, no. 8, pp. 3551-3558, Aug. 2017.

[204] A. Keshavarz and Z. Vafapour, "Sensing avian influenza viruses using terahertz metamaterial reflector," IEEE Sensors J., vol. 19, no. 13, pp. 5161-5166, 2019.

[205] A. Ren, A. Zahid, D. Fan, X. Yang, M. A. Imran, A. Alomainy, and Q. H. Abbasi, "State-of-the-art in terahertz sensing for food and water security: A comprehensive review," Trends in Food Science \& Technology, vol. 85, pp. $241-251,2019$
[206] Tuqa Khalid, Al Arabiya English, "Coronavirus: Saudi Arabian child dies after COVID-19 test swab breaks in his nose," https: //english.alarabiya.net/en/coronavirus/2020/07/15/Coronavirus-Saudichild-dies-due-to-a-COVID-19-test-swab-breaking-in-his-nose online; accessed 15 July 2020.

[207] P. R. Choudhury, "COVID-19: Researchers develop radiation technique for screening," https://news.careers360.com/covid-19-researchersdevelop-radiation-technique-for-screening online; accessed 15 July 2020.

[208] American Associates, Ben-Gurion University of the Negev, "One minute electro-optical coronavirus test developed," https://medicalxpress.com/news/2020-05-minute-electro-opticalcoronavirus.html online; accessed 15 July 2020.

[209] L. H. e. a. R. Nyberg, "5G appeal: Scientists and doctors warn of potential serious health effects of 5G," https://www.jrseco.com/wpcontent/uploads/2017-09-13-Scientist-Appeal-5G-Moratorium.pdf 09 2017, accessed: 2020-07-29.

[210] T. Caulfield, "Pseudoscience and COVID-19 - we've had enough already," Apr. 2020. [Online]. Available: https://www.nature.com/ articles/d41586-020-01266-z

[211] A. Di Ciaula, "Towards 5G communication systems: Are there health implications?" Int. J. of Hygiene and Environmental Health, vol. 221, no. 3, pp. $367-375,2018$.

[212] C. L. Russell, "5G wireless telecommunications expansion: Public health and environmental implications," Environmental Research, vol. 165, pp. $484-495,2018$.

[213] M. Simko and M.-O. Mattsson, "5G wireless communication and health effects-A pragmatic review based on available studies regarding 6 to $100 \mathrm{GHz}$," Int. J. of Environmental Research and Public Health, vol. 16, p. 3406, 092019.

[214] M. A. Jamshed, F. Héliot, and T. W. C. Brown, "A survey on electromagnetic risk assessment and evaluation mechanism for future wireless communication systems," IEEE J. of Electromagnetics, RF and Microwaves in Medicine and Biology, vol. 4, no. 1, pp. 24-36, 2020.

[215] R. Pawlak, P. Krawiec, and J. Żurek, "On measuring electromagnetic fields in 5G technology," IEEE Access, vol. 7, pp. 29 826-29 835, 2019.

[216] L. Chiaraviglio, A. S. Cacciapuoti, G. D. Martino et al., "Planning 5G networks under EMF constraints: State of the art and vision," IEEE Access, vol. 6, pp. 51 021-51037, 2018.

[217] F. H. Muhammad Ali Imran and Y. A. Sambo, Eds., Low Electromagnetic Emission Wireless Network Technologies: $5 G$ and beyond, ser. Telecommunications. Institution of Engineering and Technology, 2019. [Online]. Available: https://digital-library.theiet.org/ content/books/te/pbte084e

[218] L. Chiaraviglio, A. Elzanaty, and M.-S. Alouini, "Health risks associated with 5G exposure: A view from the communications engineering perspective," arXiv preprint, 2020. [Online]. Available: https://arxiv.org/abs/2006.00944

[219] R. A. Tell, "Broadcast radiation: How safe is safe?" IEEE Spectr, vol. 9, no. 8, pp. 43-51, 1972.

[220] S. council on electromagnetic fields, Recent Research on EMF and Health Risk: Thirteenth Report from SSM's Scientific Council on Electromagnetic Fields, ser. SSM rapport. Strålsäkerhetsmyndigheten, 2019. [Online]. Available: https://books.google.tn/books?id=Yvg9zQEACAAJ

[221] "Electromagnetic fields and public health: mobile phones," Oct. 2014, accessed: 2020-07-29. [Online]. Available: https://www.who.int/news-room/fact-sheets/detail/ electromagnetic-fields-and-public-health-mobile-phones

[222] E. I. Edeh, "Electromagnetic fields (EMFs) and public health: Shaping research agenda in the 5G tech," 08 2019, accessed: 2020-07-29. [Online]. Available: https://www.itu.int/en/ITU-T/studygroups/20172020/20/sg20rgafr/20190827/Documents/s1-p1-edeh-EMF.pdf

[223] "ICNIRP guidelines on limiting exposure to time-varying electric, magnetic and electromagnetic fields (100 kHz to $300 \mathrm{GHz})$," May. 2020, accessed: 2020-07-29. [Online]. Available: https:// www.icnirp.org/cms/upload/publications/ICNIRPrfgdl2020.pdf

[224] Scientific Committee on Emerging and Newly Identified Health Risks (SCENIHR), "Opinion on potential health effects of exposure to electromagnetic fields (emf)," https://ec.europa.eu/health/sites/ health/files/scientific_committees/emerging/docs/scenihr_o_041.pdf accessed: 2020-07-29.

[225] G. J. Wilmink and J. E. Grundt, "Current state of research on biological effects of terahertz radiation," J. of Infrared, Millimeter, and Terahertz Waves, vol. 32, no. 10, pp. 1074-1122, Oct. 2011. 
[226] A. Ramundo-Orlando and G. P. Gallerano, "Terahertz radiation effects and biological applications," J Infrared Milli Terahz. Waves, vol. 30, 08 2009.

[227] T. T. L. Kristensen, W. Withayachumnankul, P. U. Jepsen, and D. Abbott, "Modeling terahertz heating effects on water," Opt. Express, vol. 18 , no. 5, pp. 4727-4739, Mar ts

[228] B. Alexandrov, V. Gelev, A. Bishop, A. Usheva, and K. Rasmussen, "DNA breathing dynamics in the presence of a terahertz field," Physics Letters A, vol. 374, no. 10, pp. 1214 - 1217, 2010.

[229] S. M. Chitanvis, "Can low-power electromagnetic radiation disrupt hydrogen bonds in dsDNA?" J. of Polymer Science Part B: Polymer Physics, vol. 44, no. 18, pp. 2740-2747, 2006.

[230] X. Yang, X. Zhao, K. Yang, Y. Liu, Y. Liu, W. Fu, and Y. Luo, "Biomedical applications of terahertz spectroscopy and imaging," Trends in Biotechnology, vol. 34, no. 10, pp. 810-824, Oct. 2016.

[231] A. A. Angeluts, A. V. Balakin, M. G. Evdokimov et al., "Characteristic responses of biological and nanoscale systems in the terahertz frequency range," Quantum Electronics, vol. 44, no. 7, pp. 614-632, Jul. 2014.

[232] O. P. Cherkasova, V. I. Fedorov, E. F. Nemova, and A. S. Pogodin, "Influence of terahertz laser radiation on the spectral characteristics and functional properties of albumin," Optics and Spectroscopy, vol. 107, no. 4, p. 534, Nov. 2009.

[233] S. Romanenko, R. Begley, A. R. Harvey, L. Hool, and V. P. Wallace, "The interaction between electromagnetic fields at megahertz, gigahertz and terahertz frequencies with cells, tissues and organisms: Risks and potential," J. of The Royal Society Interface, vol. 14, no. 137, p. 20170585, 2017.

[234] H. Hintzsche and H. Stopper, "Effects of terahertz radiation on biological systems," Critical Reviews in Environmental Science and Technology, vol. 42, no. 22, pp. 2408-2434, 2012.

[235] S. Yamazaki, M. Harata, T. Idehara, K. Konagaya et al., "Actin polymerization is activated by terahertz irradiation," Scientific Reports, vol. 8, no. 1, p. 9990, Jul. 2018.

[236] O. P. Cherkasova, D. S. Serdyukov et al., "Effects of terahertz radiation on living cells: A review," Optics and Spectroscopy, vol. 128, no. 6, pp. 855-866, Jun. 2020.

[237] A. Doria, G. Gallerano, E. Giovenale et al., "THz radiation studies on biological systems at the ENEA FEL facility," Infrared Physics \& Technology, vol. 45, no. 5, pp. 339 - 347, 2004

[238] A. Korenstein-Ilan, A. Barbul, P. Hasin, A. Eliran, A. Gover, and R. Korenstein, "Terahertz Radiation Increases Genomic Instability in Human Lymphocytes," Radiation Research, vol. 170, no. 2, pp. $224-$ 234, 2008.

[239] G. J. Wilmink, J. E. Grundt, C. Cerna et al., "Terahertz radiation preferentially activates the expression of genes responsible for the regulation of plasma membrane properties," in Int. Conf. on Infrared, Millimeter, and Terahertz Waves, 2011, pp. 1-3.

[240] J. E. Grundt, C. Cerna, C. C. Roth et al., "Terahertz radiation triggers a signature gene expression profile in human cells," in Int. Conf. on Infrared, Millimeter, and Terahertz Waves, 2011, pp. 1-2.

[241] I. Echchgadda, J. E. Grundt, C. Z. Cerna, C. C. Roth, B. L. Ibey, and G. J. Wilmink, "Terahertz stimulate specific signaling pathways in human cells," in 39th Int. Conf. on Infrared, Millimeter, and Terahertz waves (IRMMW-THz), 2014, pp. 1-2.

[242] G. J. Wilmink, B. D. Rivest, C. C. Roth et al., "In vitro investigation of the biological effects associated with human dermal fibroblasts exposed to $2.52 \mathrm{THz}$ radiation," Lasers in Surgery and Medicine, vol. 43, no. 2, pp. 152-163, 2011.

[243] G. J. Wilmink, B. L. Ibey, C. L. Roth et al., "Determination of death thresholds and identification of terahertz $(\mathrm{THz})$-specific gene expression signatures," in Optical Interactions with Tissues and Cells XXI, E. D. Jansen and R. J. Thomas, Eds., vol. 7562, Int. Society for Optics and Photonics. SPIE, 2010, pp. $131-138$.

[244] I. Echchgadda, C. Z. Cerna, M. A. Sloan, D. P. Elam, and B. L. Ibey, "Effects of different terahertz frequencies on gene expression in human keratinocytes," in Optical Interactions with Tissue and Cells XXVI, E. D. Jansen, Ed., vol. 9321, Int. Society for Optics and Photonics. SPIE, 2015, pp. $147-155$.

[245] L. V. Titova, A. K. Ayesheshim, A. Golubov et al., "Intense THz pulses down-regulate genes associated with skin cancer and psoriasis: a new therapeutic avenue?" Scientific Reports, vol. 3, no. 1, p. 2363, Aug. 2013

[246] K.-T. Kim, J. Park, S. J. Jo et al., "High-power femtosecond-terahertz pulse induces a wound response in mouse skin," Scientific Reports, vol. 3, no. 1, p. 2296, Aug. 2013
[247] J. Olshevskaya, A. Kozlov, A. K. Petrov, T. Zapara, and A. S. Ratushnyak, "Influence of terahertz (submillimeter) laser radiation on cell membrane permeability," Vestnik Novosibirsk State University. Series: Physics, vol. 5, pp. 185-189, 012010.

[248] T. A. Zapara, S. P. Treskova, and A. S. Ratushniak, "Effect of antioxidants on the interaction of terahertz (submillimeter) laser radiation and neuronal membrane," J. of Surface Investigation. X-ray, Synchrotron and Neutron Techniques, vol. 9, no. 5, pp. 869-871, Sep. 2015.

[249] M. Borovkova, M. Serebriakova, V. Fedorov et al., "Investigation of terahertz radiation influence on rat glial cells," Biomed. Opt. Express, vol. 8, no. 1, pp. 273-280, Jan. 2017.

[250] A. A. Angeluts, A. B. Gapeyev, M. N. Esaulkov et al., "Study of terahertz-radiation-induced DNA damage in human blood leukocytes," Quantum Electronics, vol. 44, no. 3, pp. 247-251, mar 2014.

[251] O. Zeni, G. P. Gallerano, A. Perrotta et al., "Cytogenetic observations in human peripheral blood leukocytes following in vitro exposure to THz radiation: A pilot study," Health physics, vol. 92, pp. 349-57, 05 2007.

[252] O. Cherkasova, M. Surovtseva, A. Lykov, O. Kazakov et al., "Studying the effect of $0.14 \mathrm{THz}$ radiation on human dermal fibroblasts," AIP Conf. Proc., vol. 2098, no. 1, p. 020004, 2019.

[253] N. Yaekashiwa, S. Otsuki, S. Hayashi, and K. Kawase, "Investigation of the non-thermal effects of exposing cells to $70-300 \mathrm{GHz}$ irradiation using a widely tunable source," J. of Radiation Research, vol. 59, no. 2, pp. 116-121, 122017.

[254] J. S. Olshevskaya, A. S. Ratushnyak, A. K. Petrov, A. S. Kozlov, and T. A. Zapara, "Effect of terahertz electromagnetic waves on neurons systems," in IEEE Region 8 Int. Conf. on Computational Technologies in Electrical and Electronics Engineering, 2008, pp. 210-211.

[255] A. Abohmra, H. Abbas, S. F. Jilani, A. Alomainy, M. A. Imran, and Q. H. Abbasi, "High bandwidth Perovskite based antenna for highresolution biomedical imaging at Terahertz," in IEEE Int. Sym. on Antennas and Propag. and USNC-URSI Radio Sci. Meet., 2019, pp. 503-504.

[256] K. Yang, D. Bi, Y. Deng, R. Zhang, M. M. U. Rahman, N. A. Ali, M. A. Imran, J. M. Jornet, Q. H. Abbasi, and A. Alomainy, "A comprehensive survey on hybrid communication for internet of nanothings in context of body-centric communications," arXiv preprint, Dec. 2019. [Online]. Available: https://arxiv.org/abs/1912.09424

[257] H. Sarieddeen, M. Alouini, and T. Y. Al-Naffouri, "Terahertz-band ultra-massive spatial modulation MIMO," vol. 37, no. 9, pp. 20402052, 2019.

[258] A. Faisal, H. Sarieddeen, H. Dahrouj, T. Y. Al-Naffouri, and M.-S. Alouini, "Ultra-massive MIMO systems at terahertz bands: Prospects and challenges," arXiv preprint, 2019. [Online]. Available: https://arxiv.org/abs/1902.11090

[259] M. H. Loukil, H. Sarieddeen, M.-S. Alouini, and T. Y. Al-Naffouri, "Terahertz-band mimo systems: Adaptive transmission and blind parameter estimation," 2019

[260] A. Vavouris, F. Dervisi, V. Papanikolaou, P. Diamantoulakis, G. Karagiannidis, and S. Goudos, "An energy efficient modulation scheme for body-centric Terahertz (THz) nanonetworks," Technologies, vol. 7, no. 1, p. 14, Jan. 2019.

[261] L. Feng, A. Ali, M. Iqbal, A. K. Bashir, S. A. Hussain, and S. Pack, "Optimal haptic communications over nanonetworks for E-Health systems," IEEE Trans. Ind. Informat., vol. 15, no. 5, pp. 3016-3027, 2019.

[262] N. Saeed, H. Nam, T. Y. Al-Naffouri, and M.-S. Alouini, "A stateof-the-art survey on multidimensional scaling-based localization techniques," IEEE Commun. Surveys Tuts., vol. 21, no. 4, pp. 3565-3583, 2019.

[263] W. Aman, M. M. U. Rahman, H. T. Abbas, M. A. Khalid, M. A. Imran, A. Alomainy, and Q. H. Abbasi, "Securing the insecure: A first-line-of-defense for nanoscale communication systems operating in THz band," arXiv preprint, Jul. 2020. [Online]. Available: https://arxiv.org/abs/2007.06818 